\title{
Antiferromagnetic, Neutral, and Superconducting Band in $\mathrm{La}_{2} \mathrm{CuO}_{4}$
}

\author{
Ekkehard Krüger* \\ Max-Planck-Institut für Metallforschung, D-70506 Stuttgart, Germany
}

(Dated: September 5, 2018)

\begin{abstract}
The symmetry of the Bloch functions in the conduction band of tetragonal and orthorhombic $\mathrm{La}_{2} \mathrm{CuO}_{4}$ is examined for the existence of symmetry-adapted and optimally localizable (usual or spin-dependent) Wannier functions. It turns out that such Wannier functions do not exist in the tetragonal phase. In the orthorhombic phase, on the other hand, the Bloch functions can be unitarily transformed in three different ways into optimally localizable Wannier functions: they can be chosen to be adapted to each of the three phases observed in the pure or doped material, that is, to the antiferromagnetic phase, to the superconducting phase or to the phase evincing neither magnetism nor superconductivity. This group-theoretical result is proposed to be interpreted within a nonadiabatic extension of the Heisenberg model. Within this model, atomiclike states represented by these Wannier functions are responsible for the stability of each of the three phases. However, all the three atomiclike states cannot exist in the tetragonal phase, but are stabilized by the orthorhombic distortion of the crystal. A simple model is proposed which might explain the physical properties of $\mathrm{La}_{2-x} \mathrm{Sr}_{x} \mathrm{CuO}_{4}$ as a function of the $\mathrm{Sr}$ concentration $x$.
\end{abstract}

Keywords: superconductivity, magnetism, Heisenberg model, group theory

\section{INTRODUCTION}

\section{1. $\mathrm{La}_{2-x} \mathrm{Sr}_{x} \mathrm{CuO}_{4}$}

The physical properties of pure and doped $\mathrm{La}_{2-x} \mathrm{Sr}_{x} \mathrm{CuO}_{4}$ have been studied in a broad range of $\mathrm{Sr}$ concentrations $x[1,2]$. Pure $\mathrm{La}_{2} \mathrm{CuO}_{4}$ is antiferromagnetic below about $300 \mathrm{~K}$, but this magnetic long-range order disappears at a $\mathrm{Sr}$ concentration of $x \approx 0.015$. Between $x \approx 0.015$ and $x \approx 0.05$ the samples evince neither an antiferromagnetic or a superconducting state. This border region has features characteristic of spin-glass systems [3]. Finally, above $x \approx 0.05$ the material becomes a high- $T_{c}$ superconductor.

Furthermore, $\mathrm{La}_{2} \mathrm{CuO}_{4}$ undergoes a very interesting structural phase transition at about $520 \mathrm{~K}$ from a hightemperature tetragonal phase to a low-temperature orthorhombic phase [1]. The tetragonal structure with the space group $I 4 / \mathrm{mmm}$ is depicted in Fig. 1. The major change in the structure in the tetragonal to orthorhombic transition involves a "buckling of the copper-oxygen planes" [2]: in the orthorhombic phase with the space group $\mathrm{Cmca}$ the oxygen atoms are slightly shifted from their tetragonal positions in such a way that the translation $\vec{t}_{3}$ in Fig. 1 is doubled $[1,2]$, see Fig. 2. The orthorhombic distortion is so small that the orthorhombic and tetragonal structures are not distinguishable on the scale of the figures [2].

The buckling of the copper-oxygen planes has been established also in doped $\mathrm{La}_{2-x} \mathrm{Sr}_{x} \mathrm{CuO}_{4}$ for a broad range of $\mathrm{Sr}$ concentrations $x$. At zero temperature, is exists up to $x \approx 0.2$ and disappears for higher $\mathrm{Sr}$ concentrations [1].

*Electronic address: krueger@mf .mpg.de

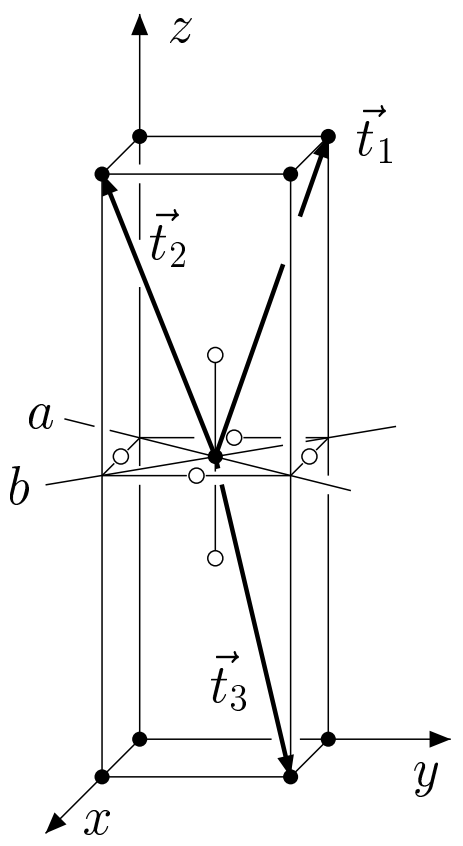

FIG. 1: Structure of tetragonal $\mathrm{La}_{2} \mathrm{CuO}_{4}$, showing the basic translations of the Bravais lattice $\Gamma_{q}^{v}$. The space group is $I 4 / \mathrm{mmm}$. Open circles are $\mathrm{O}$, solid are $\mathrm{Cu}$. La is not shown.

\subsection{Magnetic and superconducting bands}

An energy band is called "magnetic band with the magnetic group $M$ " if the Bloch functions of this band can be unitarily transformed into Wannier functions which are

- centered on the atomic positions; 


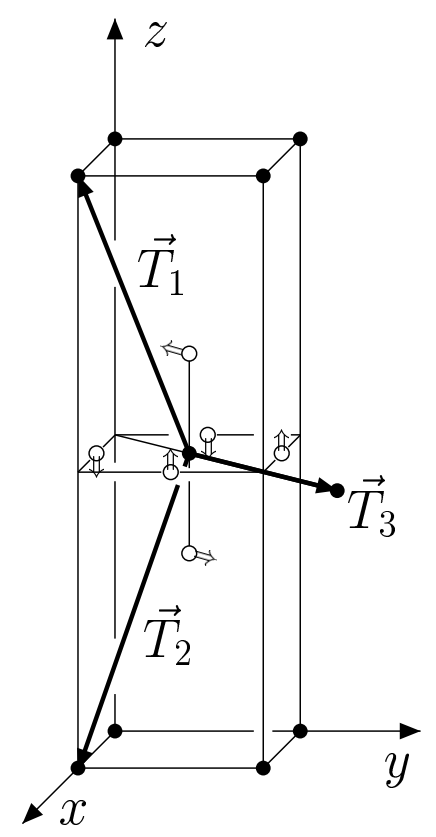

FIG. 2: Structure of orthorhombic $\mathrm{La}_{2} \mathrm{CuO}_{4}$ with the space group $\mathrm{Cmca}$, showing the basic translations of the Bravais lattice $\Gamma_{o}^{b}$. Open circles are $\mathrm{O}$, solid are $\mathrm{Cu}$. La is not shown. The arrows $\Uparrow$ indicate the distortions of the $\mathrm{O}$ atoms.

- symmetry-adapted to $M$; and

- optimally localizable.

"Optimally localizable" means that the Wannier functions are as well localizable as possible. Observations on the band structures of $\mathrm{Fe}[4]$ and $\mathrm{Cr}$ [5] suggest that in both $\mathrm{Fe}$ and $\mathrm{Cr}$ a narrow, roughly half-filled magnetic band is responsible for the stability of the ferromagnetic and the (antiferromagnetic) spin-density wave state, respectively, in these materials. This observation can be interpreted within a nonadiabatic extension of the Heisenberg model, the "nonadiabatic Heisenberg model" (NHM) [6].

An energy band is called "superconducting band" if the Bloch functions of this band can be unitarily transformed into spin-dependent Wannier functions which are

- centered on the atomic positions;

- symmetry-adapted to the space group of the considered material; and

- optimally localizable.

Observations on the band structures of a great number of elemental metals suggest that narrow, roughly half-filled superconducting bands are responsible for the stability of superconducting states. Also this observation can be interpreted within the NHM [7-9].
Optimally localizable and symmetry-adapted Wannier functions are defined in Appendix B. The small irreducible representations of all the magnetic or superconducting energy bands in the space groups of $\mathrm{La}_{2} \mathrm{CuO}_{4}$ are listed in Tables XI - XVI at the end of this paper. The way to determine these symmetry labels is also summarized in Appendix B.

\subsection{Nonadiabatic Heisenberg model}

Within the NHM, the electrons in narrow, half-filled bands may lower their Coulomb energy by occupying an atomiclike state as defined by Mott [10] and Hubbard [11]: the electrons occupy as long as possible localized states and perform their band motion by hopping from one atom to another. The Hamiltonian $H^{n}$ within the NHM is distinguished from any adiabatic Hamiltonian by a particular feature: the symmetry of $H^{n}$ depends on the symmetry of the localized functions representing the localized states. As a consequence, within this model the localized states must be represented by optimally localizable Wannier functions which are adapted to the symmetry of the considered phase. These Wannier functions may be usual (spin-independent) or spin-dependent Wannier functions.

Spin-independent Wannier functions are related to magnetic bands, and, for the first time in this paper, to a "neutral" band. In an atomiclike state represented by spin-dependent Wannier functions, the conservation of crystal spin requires a strong coupling between the electron spins and the crystal spins of suitable boson excitations. Below a transition temperature, this spin-boson interaction leads to stable Cooper pairs.

The mechanism of Cooper pair formation within such an atomiclike system resembles the familiar BCS mechanism [12]. However, the Cooper pairs are stabilized in a new way by constraining forces. Though these constraining forces neither are considered within the original BCS theory nor within the modern theory of superconductivity, it cannot be excluded that they must operate in superconducting states because electrons forming Cooper pairs possess only half of the degrees of freedom of free electrons.

The above mentioned "suitable bosons" are boson excitations bearing the crystal-spin angular momentum $1 \cdot \hbar$ and being sufficiently stable to carry it through the crystal. The order of magnitude of the superconducting transition temperature is determined by the excitation energy of those stable crystal-spin-1 bosons of the crystal which have the lowest excitation energy [13].

All the Wannier functions considered in this paper are centered at the $\mathrm{Cu}$ atoms because $\mathrm{La}_{2} \mathrm{CuO}_{4}$ has a single conduction band $[6]$. 


\section{CONDUCTION BAND OF TETRAGONAL $\mathrm{La}_{2} \mathrm{CuO}_{4}$}

Tetragonal $\mathrm{La}_{2} \mathrm{CuO}_{4}$ possesses a pronounced conduction band characterized by the small representations

$$
Z_{1}^{+}, \Gamma_{2}^{-}, X_{3}^{+}, P_{3} \text {, and } N_{1}^{+},
$$

see Fig. 3.

\subsection{Exclusion of a neutral atomiclike state with usual Wannier functions}

Band (1) is not listed in Table XI. Hence, in the tetragonal phase of $\mathrm{La}_{2} \mathrm{CuO}_{4}$ the electrons of this band cannot lower their Coulomb energy by occupying an atomiclike state because suitable symmetry-adapted and optimally localizable Wannier functions do not exist.

\subsection{Exclusion of a superconducting band}

Taking into account the electron spin, the conduction band (1) is characterized by the double-valued small representations

$$
Z_{6}^{+}, \Gamma_{6}^{-}, X_{5}^{+}, P_{7}, \text { and } N_{3}^{+}+N_{4}^{+},
$$

see Table IX. This band is not a superconducting band because it is not listed in Table XII. Hence, the electrons of this band cannot lower their Coulomb energy by occupying an atomiclike state even if the Wannier functions representing the localized states are allowed to be spin-dependent. Thus, in the framework of the NHM we cannot expect stable Cooper pairs in the tetragonal phase of $\mathrm{La}_{2} \mathrm{CuO}_{4}$.

\section{EXCLUSION OF AN ANTIFERROMAGNETIC BAND WITH THE SPACE GROUP OF ANTIFERROMAGNETIC CHROMIUM}

Folding the conduction band (1) into the Brillouin zone of antiferromagnetic $\mathrm{Cr}$ (as given, e.g., in Fig. 3 of Ref. [15]), the band becomes characterized by the small representations

$$
\Gamma_{2}^{+}+\Gamma_{2}^{-}, M_{20}, A_{10}, \text { and } R_{5},
$$

see Table VII. This band is not listed in Table XV. Hence, optimally localizable Wannier functions being adapted to $P 4 / m n c$ do not exist. The electrons cannot lower their Coulomb energy by occupying an atomiclike state represented by such antiferromagnetic Wannier functions. Hence, a spin structure with the space group $P 4 / m n c$ (that means, with the spins lying parallel to the $z$ axis in Fig. 1) is not predicted within the NHM.

\section{CONDUCTION BAND OF ORTHORHOMBIC $\mathrm{La}_{2} \mathrm{CuO}_{4}$}

In the tetragonal phase of $\mathrm{La}_{2} \mathrm{CuO}_{4}$, it is the symmetry of the Bloch functions at point $\Gamma$ which does not allow the construction of symmetry-adapted and optimally localizable (spin-dependent) Wannier functions: the Bloch functions at $\Gamma$ are negative with respect to the inversion, but at the other points of symmetry they are positive.

In the orthorhombic phase of $\mathrm{La}_{2} \mathrm{CuO}_{4}$ the oxygen atoms are slightly displaced from their tetragonal positions [2], see Fig. 2. In the Brillouin zone of this phase, the tetragonal points $\Gamma$ and $X$ become equivalent, see Fig. 4. Hence, in the orthorhombic Brillouin zone we may replace the unsuited Bloch functions at the tetragonal point $\Gamma$ by suited Bloch functions coming from the tetragonal point $X$, if they exist. In fact, there exist well-suited functions at the tetragonal point $X$ which are positive with respect to the inversion and have nearly the same energy as the functions at the tetragonal points $\Gamma$ and $Z$. These are the Bloch functions with $X_{2}^{+}$and $X_{4}^{+}$ symmetry, see Fig. 3.

We get the "active band" denoted in Fig. 5 by the bold line if we replace the tetragonal $Z_{1}^{+}$and $\Gamma_{2}^{-}$functions by the tetragonal $X_{2}^{+}$and $X_{4}^{+}$functions. This band is proposed to be responsible for the physical properties of $\mathrm{La}_{2} \mathrm{CuO}_{4}$. Symmetry-adapted Wannier functions constructed from the Bloch functions of the active band represent localized states with orthorhombic symmetry. However, we may assume that these orthorhombic states do not strongly differ from states with tetragonal symmetry, i.e., we may assume that these orthorhombic states are states of slightly distorted tetragonal symmetry. This is because, first, the predominant portion of the Bloch functions of this band comes from the Bloch functions of the initial tetragonal band (1), and, second, the new tetragonal $X$ functions have nearly the same energy as the initial tetragonal $Z$ and $\Gamma$ functions.

The active band in the orthorhombic structure is characterized by the representations

$$
\Gamma_{2}^{+}+\Gamma_{3}^{+}, Y_{2}^{+}+Y_{3}^{+}, R_{1}^{+}+R_{2}^{+}, T_{2} \text { and } Z_{2},
$$

where $\Gamma_{2}^{+}$comes from the tetragonal $X_{2}^{+}$state, $\Gamma_{3}^{+}$comes from $X_{3}^{+}, Y_{2}^{+}$comes from $X_{4}^{+}, Y_{3}^{+}$comes from $X_{3}^{+}$, too, $R_{1}^{+}+R_{2}^{+}$comes from $N_{1}^{+}$, and $T_{2}$ and $Z_{2}$ come from states at the tetragonal $U$ and $\Delta$ lines, respectively, see Table VI.

Band (4) is identical to band 2 in Table XIV. Hence, the Bloch functions of this band can be unitarily transformed into optimally localizable Wannier functions symmetry-adapted to the space group $\mathrm{Cmca}$. The electrons of this band may lower their Coulomb energy by occupying an atomiclike state represented by these Wannier functions. However, the related nonadiabatic Hamiltonian $H^{n}$ has no longer the symmetry of the tetragonal space group $I 4 / \mathrm{mmm}$ since it commutes only with those space group operators of the tetragonal group $I 4 / \mathrm{mmm}$ which also belong to the subgroup Cmca. Therefore, 


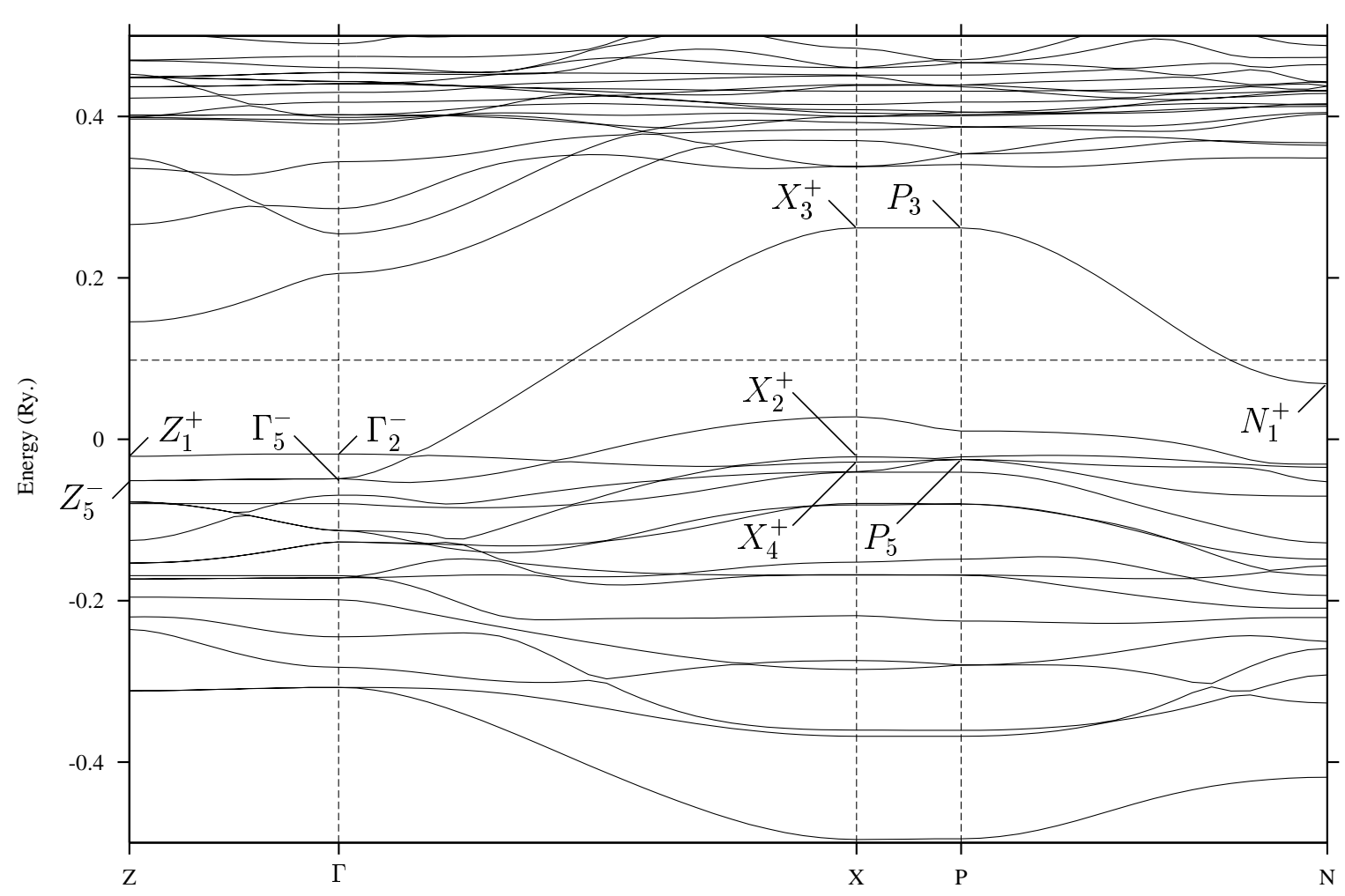

FIG. 3: Band structure of tetragonal $\mathrm{La}_{2} \mathrm{CuO}_{4}$ as calculated by Ove Jepsen [14], with symmetry labels determined by the author. The band characterized by $Z_{1}^{+}, \Gamma_{2}^{-}$(or $Z_{5}^{-}, \Gamma_{5}^{-}$), $X_{3}^{+}+X_{2}^{+}, P_{3}+P_{5}$, and $N_{1}^{+}$forms a natural narrow conduction band. However, the electrons of this nearly half-filled band cannot occupy the (energetically favorable) atomiclike state because suitable symmetry-adapted and optimally localizable Wannier functions do not exist in the tetragonal phase. However, when the bands are folded into the Brillouin zone of the orthorhombic phase (see Fig. 5), the points $X$ and $\Gamma$ become equivalent. In the new Brillouin zone we may replace the $Z_{1}^{+}$and $\Gamma_{2}^{-}$Bloch functions by the $X_{2}^{+}$and $X_{4}^{+}$functions of nearly the same energy. With these new functions, the construction of symmetry-adapted Wannier becomes possible.

such an atomiclike state represented by Wannier functions of reduced symmetry requires a change of the symmetry of the crystal.

\subsection{Antiferromagnetic band with the orthorhombic space group Cmca}

The space group Cmca is the space group of the antiferromagnetic structure depicted in Fig. 6. Thus, the electrons might occupy an atomiclike state by putting their spins antiparallel as shown in Fig. 6. This appears to be possible, because the Wannier functions of band 2 in Table XIV can be constructed in such a way that they are symmetry-adapted to the whole magnetic group

$$
M=C m c a+\left\{K \mid \frac{1}{2} \frac{1}{2} \frac{1}{2}\right\} C m c a,
$$

where $K$ denotes the operator of time-inversion, see Appendix B. In this case, no spatial distortion of the tetragonal lattice would be required, since the spin structure by itself together with the accompanying magnetostriction represents a change of the tetragonal symmetry.
However, within the NHM stable spin structures with the space group $\mathrm{Cmca}$ do not exist because $M$ does not possess suitable corepresentations, see Appendix A.

\subsection{Spatially distorted crystal with the orthorhombic space group $\mathrm{Cmca}$}

Hence, the electrons can occupy the energetically favorable atomiclike state only in a spatially distorted crystal. Therefore, the system produces the experimentally established distortion with the space group $\mathrm{Cmca}$. This is possible because of the favorable position of the oxygen atoms: suitable displacements of these atoms also produce a crystal with the antiferromagnetic space group Cmca. Within the distorted system, there exist three different atomiclike states. 


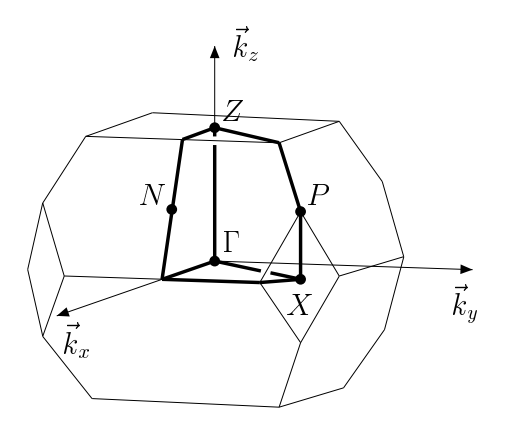

4.2.2. Antiferromagnetic band with the space group Pccn of
the experimentally established antiferromagnetic structure

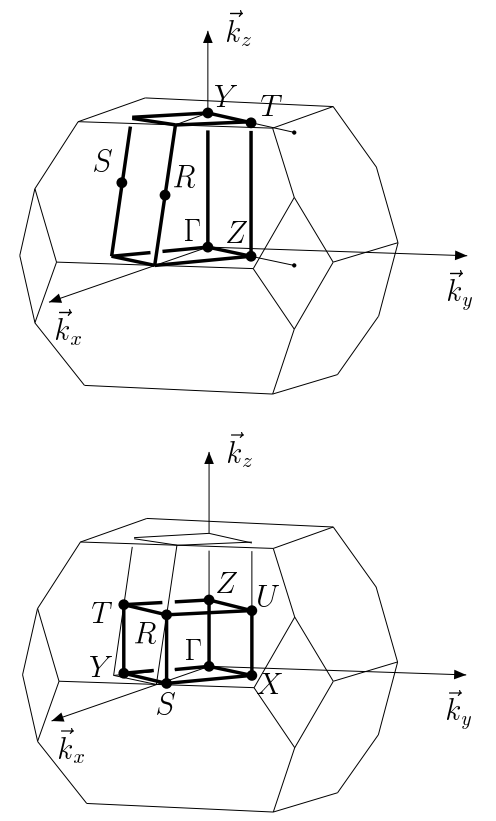

The experimentally established [1] antiferromagnetic structure of $\mathrm{La}_{2} \mathrm{CuO}_{4}$ with the space group Pccn is depicted in Fig. 7. Folding the active band (4) into the Brillouin zone of Pccn (given in Fig. 4), we get a band consisting of four branches which is characterized by the representations

$$
\begin{gathered}
\Gamma_{1}^{+}+\Gamma_{2}^{+}+\Gamma_{3}^{+}+\Gamma_{4}^{+}, R_{1}^{+}+R_{2}^{+}+R_{3}^{+}+R_{4}^{+}, \\
Y_{1}+Y_{2}, X_{1}+X_{2}, Z_{1}+Z_{2} \\
U_{1}+U_{2}, T_{1}+T_{2}, \text { and } S_{1}+S_{2}
\end{gathered}
$$

see Table VIII. This band is identical to band 1 in Table XVI. Hence, the Bloch functions of this band can be unitarily transformed into optimally localizable Wannier functions symmetry-adapted to the space group Pccn. Thus, within the NHM the electrons may lower their Coulomb energy by producing the antiferromagnetic structure shown in Fig. 7. However, this spin structure is connected with the experimentally established spatial distortion of the tetragonal lattice because the spin structure alone does not produce a crystal with the space group Pccn.

Fig. 7 suggests that the related magnetic group may either be written as

$$
M=P c c n+\left\{K \mid \frac{1}{2} \frac{1}{2} 0\right\} P c c n
$$

or

$$
M=P c c n+\left\{K \mid \frac{1}{2} 0 \frac{1}{2}\right\} P c c n,
$$

where still $K$ denotes the operator of time-inversion.

The first magnetic group (8) possesses suitable corep-

FIG. 4: Brillouin zones of $\mathrm{La}_{2} \mathrm{CuO}_{4}$.

Top: Brillouin zone of the tetragonal space group $I 4 / \mathrm{mmm}$ with the Bravais lattice $\Gamma_{q}^{v}$, as given in Fig. 3.10 (b) of Ref. [16].

Center: Basic domain of the Brillouin zone of the orthorhombic space group $C m c a$ with the Bravais lattice $\Gamma_{o}^{b}$ within the Brillouin zone of $\Gamma_{q}^{v}$.

Bottom: Basic domain of the Brillouin zone of the space group Pccn of the antiferromagnetic phase with the Bravais lattice $\Gamma_{o}$ within the basic domain of the Brillouin zone of $\Gamma_{o}^{b}$.

\subsubsection{Neutral band}

The Wannier functions of band 2 in Table XIV may also be constructed so that they are symmetry-adapted to the grey magnetic group

$$
M=C m c a+K \cdot C m c a \text {. }
$$

Thus, in this spatially distorted crystal the conduction electrons can occupy a "neutral" atomiclike state represented by these spin-independent Wannier functions being invariant under time-inversion. I believe that this neutral state is responsible for the phase of $\mathrm{La}_{2-x} \mathrm{Sr}_{x} \mathrm{CuO}_{4}$ which is neither antiferromagnetic nor superconducting.

resentations and, hence, spin structures with this group can be stable within the NHM, see Appendix A.

\subsubsection{Superconducting band}

Taking into account the electron spin, the active band (4) is characterized by the double-valued representations

$$
2 \Gamma_{5}^{+}, 2 Y_{5}^{+}, 2 R_{3}^{+}+2 R_{4}^{+}, T_{3}+T_{4} \text { and } Z_{3}+Z_{4},
$$

see Table $\mathrm{X}$. This band is identical to band 1 in Table XIII. Hence, the Bloch functions of this band can be unitarily transformed into optimally localizable spin-dependent Wannier functions symmetry-adapted to the space group Cmca. The electrons may lower their Coulomb energy by occupying an atomiclike state represented by these spin-dependent Wannier functions. In this case, the conservation of crystal spin requires a strong coupling between the electron spins and the crystal spins of suitable boson excitations. Below a transition temperature, this coupling leads to a superconducting state. However, this superconducting state exists only in the space group Cmca and hence, is connected with the experimentally established spatial distortion of the tetragonal lattice. 


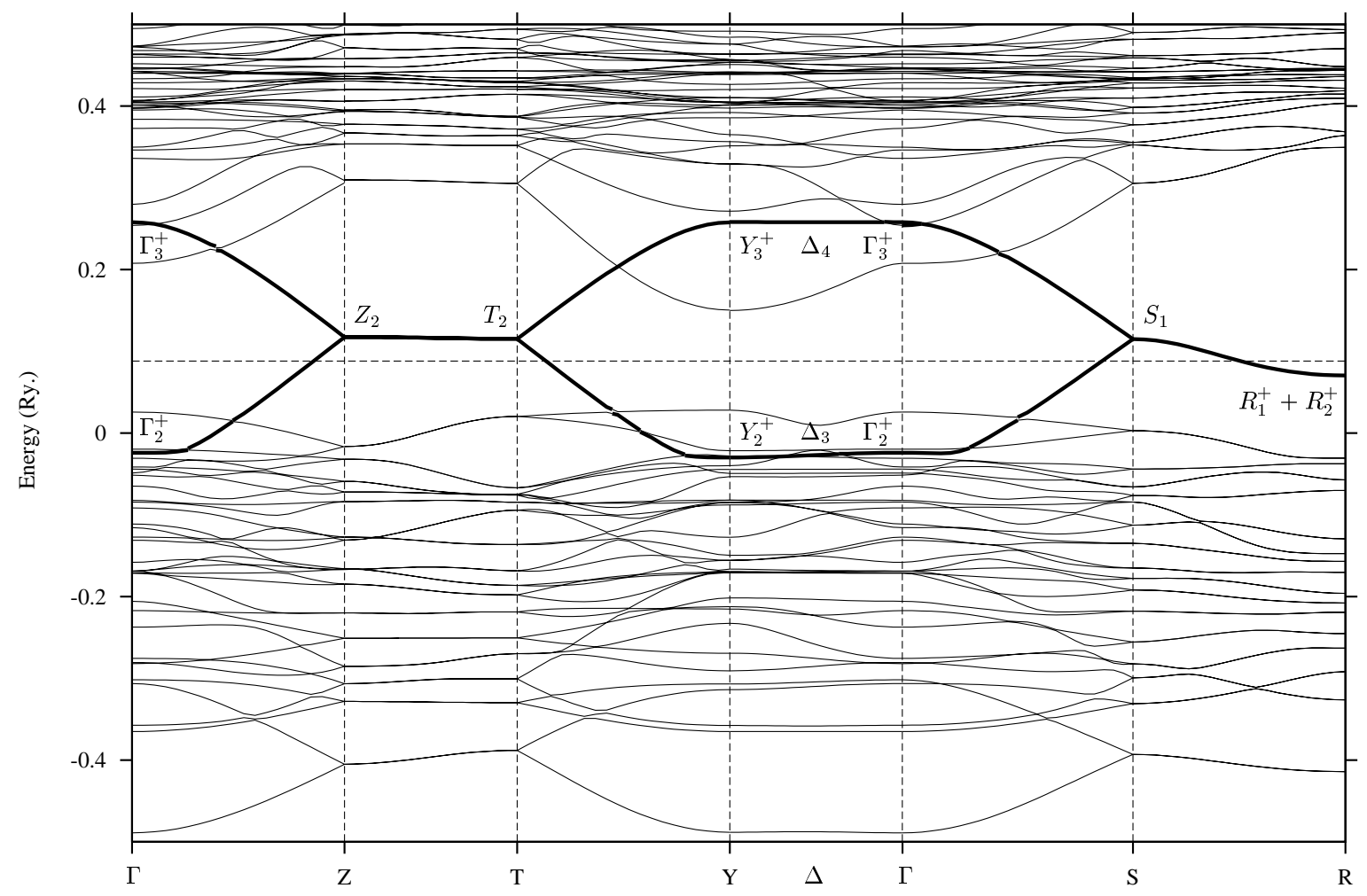

FIG. 5: Band structure of orthorhombic $\mathrm{La}_{2} \mathrm{CuO}_{4}$ as calculated by Ove Jepsen [14], with symmetry labels determined by the author. The bold line shows the "active band" which is proposed to be responsible for the physical properties of $\mathrm{La}_{2} \mathrm{CuO}_{4}$. The electrons of this band could occupy an energetically favorable atomiclike state by putting their spins antiparallel as shown in Fig. 6. Such a spin structure with the space group $\mathrm{Cmca}$ would not require any distortion of the tetragonal lattice. However, this spin structure is not stable because the related magnetic group does not possess suitable corepresentations. Alternatively, the electrons may occupy an atomiclike state either by producing the experimentally established spin structure with the space group Pccn, a "neutral" atomiclike state invariant under time-inversion (and hence, without any spin structure) or a superconducting state, all of them being stable only in a (slightly) distorted lattice with the orthorhombic space group $C m c a$.

\section{SUMMARY AND DISCUSSION}

\subsection{Pure $\mathrm{La}_{2} \mathrm{CuO}_{4}$}

The symmetry of the optimally localizable Wannier functions in the conduction band of $\mathrm{La}_{2} \mathrm{CuO}_{4}$ suggests that some aspects of the physical properties of this compound can be understood within the NHM in terms of magnetic, neutral, and superconducting bands.

The conduction band of tetragonal $\mathrm{La}_{2} \mathrm{CuO}_{4}$ is clearly an "antiferromagnetic" band tending to produce the antiferromagnetic structure with the space group Cmca illustrated in Fig. 6. The conduction electrons would be able to occupy an atomiclike state by producing this spin structure without any additional spatial distortion of the tetragonal crystal. However, the nonadiabatic Hamiltonian $H^{n}$ does not possess antiferromagnetic eigenstates with the space group $\mathrm{Cmca}$ because the related magnetic group has no suitable corepresentations.

Thus, the system is forced to produce a spatial distortion of the crystal so the conduction electrons can occupy the energetically favorable atomiclike state. We recognize the intention of the nature to stabilize the atomiclike state with the least possible distortion: the positions of the oxygen atoms are shifted in a subtle manner in order that the basic translation $\vec{t}_{3}$ (see Fig. 1) in the tetragonal lattice becomes doubled while the other two basic translations $\vec{t}_{1}$ and $\vec{t}_{2}$ still connect two lattice points. This slightly distorted crystal has the experimentally established orthorhombic space group Cmca, that is, the same space group as the spin structure in Fig. 6. Within the distorted system, the conduction electrons now have three possibilities to condense into an atomiclike state.

First, in the orthorhombic crystal the Bloch functions of the conduction band can be unitarily transformed into optimally localizable Wannier functions adapted to the symmetry of the experimentally established spin structure (depicted in Fig. 7) with the space group Pccn. In this case, the related magnetic group [given in Eq. (8)] possesses suitable corepresentations in order that $H^{n}$ has eigenstates with this spin structure. Therefore, the electrons may occupy the energetically favorable atomi- 


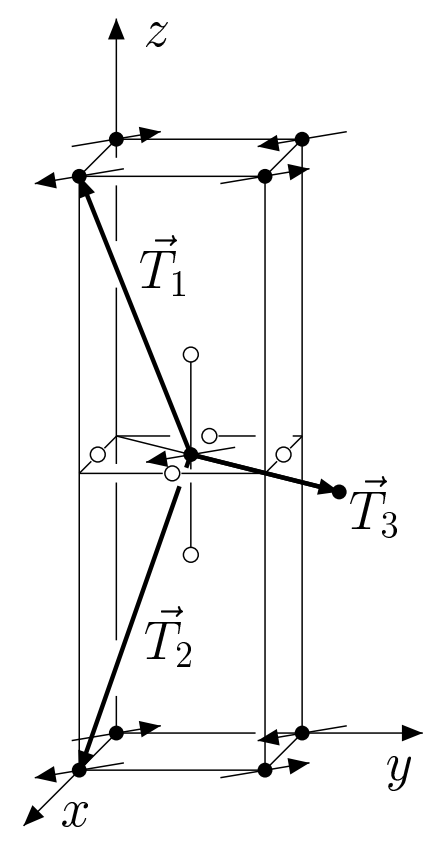

FIG. 6: Hypothetical spin structure of $\mathrm{La}_{2} \mathrm{CuO}_{4}$ with the space group $\mathrm{Cmca}$. However, this spin structure is not stable because the related magnetic group does not possess suitable corepresentations.

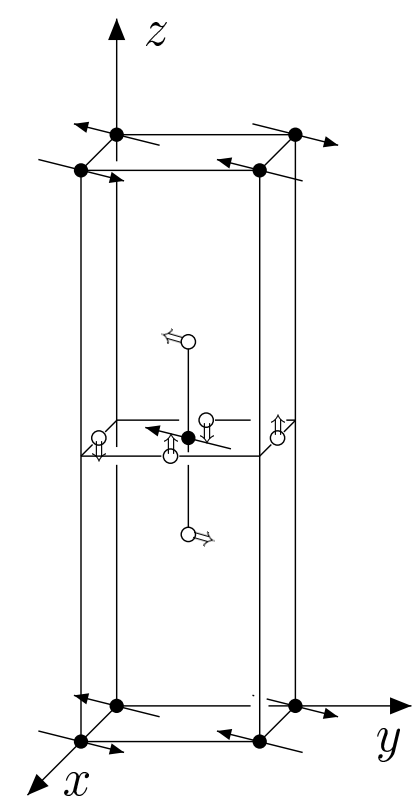

FIG. 7: Experimentally established [1] spin structure of $\mathrm{La}_{2} \mathrm{CuO}_{4}$ with the space group Pccn. Open circles are O, solid are $\mathrm{Cu}$. La is not shown. The arrows $\Uparrow$ indicate the distortions of the $\mathrm{O}$ atoms.

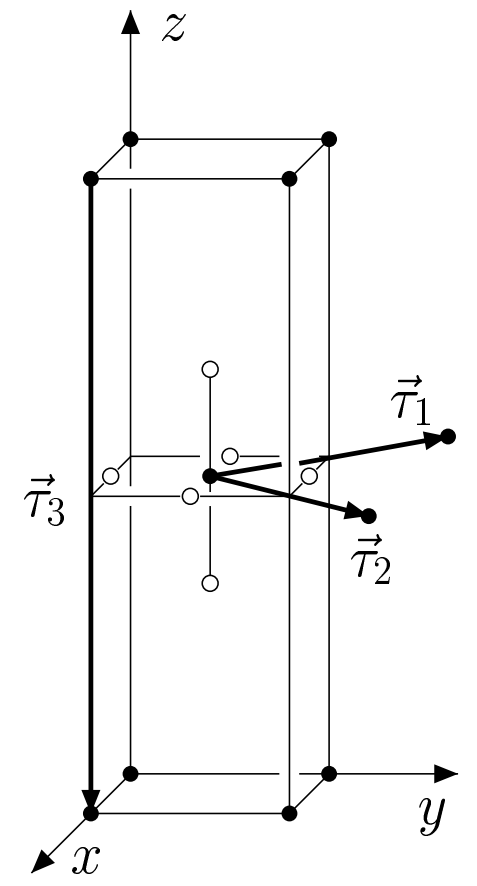

FIG. 8: Basic translations of the Bravais lattice $\Gamma_{o}$ of the experimentally established spin structure of $\mathrm{La}_{2} \mathrm{CuO}_{4}$ shown in Fig. 7.

clike state by producing this antiferromagnetic structure. However, it is the spatial orthorhombic distortion of the crystal which stabilizes the antiferromagnetic structure.

Secondly, the orthorhombic distortion of the crystal need not be accompanied by a spin structure. In the distorted system there exist optimally localizable (usual) Wannier functions which are adapted to the orthorhombic symmetry. I believe that the "neutral" atomiclike state represented by these Wannier functions is responsible for the "spin-glass" phase [1] of doped $\mathrm{La}_{2} \mathrm{CuO}_{4}$ because this phase neither is antiferromagnetic nor is superconducting. However, the question why such a neutral state has features of spin-glass systems [3], requires further investigation. Also in this case, it is the experimentally established orthorhombic distortion which stabilizes the neutral phase.

Thirdly, in the orthorhombic system the conduction electrons may occupy an atomiclike state represented by spin-dependent Wannier functions. In such an atomiclike state, the conservation of crystal spin requires a strong coupling between the electron spins and the crystal spins of stable crystal-spin-1 boson excitations. Below a transition temperature $T_{c}$, this spin-boson interaction leads to the formation of Cooper pairs. Also this spin-boson phase is not stable in the tetragonal system but is stabilized by the orthorhombic distortion.

Most likely, in any material the stable crystal-spin-1 bosons of lowest excitation energy are coupled phonon- 
plasmon modes. In the isotropic lattices of the transition elements they have dominant phonon character. However, phonon-like excitations are not able to transport crystal-spin angular-momenta within the two-dimensional copper-oxygen layers of $\mathrm{La}_{2} \mathrm{CuO}_{4}$ [13]. Thus, in the spin-boson phase the electron spins interact with boson excitations of dominant plasmon character. The relatively high energy of these plasmon-like crystalspin-1 bosons is proposed to be responsible for the high superconducting transition temperature observed in the doped material.

\subsection{Doped $\mathrm{La}_{2-x} \mathrm{Sr}_{x} \mathrm{CuO}_{4}$}

In doped $\mathrm{La}_{2-x} \mathrm{Sr}_{x} \mathrm{CuO}_{4}$ the symmetry of the crystal is disturbed and all the statements in the preceding section are, strictly speaking, no longer valid. However, we may assume that for small values of $x$ the given description of the atomiclike states holds also in the doped system because the symmetry of the physically relevant copperoxygen layers is not seriously affected by the doping.

In doped $\mathrm{La}_{2-x} \mathrm{Sr}_{x} \mathrm{CuO}_{4}$, all three possible atomiclike states described in the preceding section are evidently realized: for $0 \leq x<0.015$ the antiferromagnetic state has the lowest energy, then a neutral phase is stable and above $x \approx 0.05$ the material becomes superconducting [1]. I propose a simple model which might help to understand this $x$ dependence of the physical properties of $\mathrm{La}_{2-x} \mathrm{Sr}_{x} \mathrm{CuO}_{4}$ :

At the transition from the adiabatic to the nonadiabatic system (i.e., at the transition from a purely bandlike to the atomiclike state), the total energy of the electron system decreases by the "condensation energy"

$$
\Delta E_{i}(x)=\Delta E_{i}^{e}-\Delta E_{i}^{d}(x),
$$

where $i=1,2$, and 3 labels the antiferromagnetic, the neutral, and the spin-boson phase, respectively. (Below the transition temperature, the latter phase is superconducting.)

$\Delta E_{i}^{e}$ stands for the electronic part of the condensation energy. It is defined in Eqs. (2.20) and (5.3) of Ref. [6] for the superconducting and magnetic state, respectively, and may be assumed to be independent of the Sr concentration $x$ since the physically relevant copper-oxygen planes are not seriously affected by the doping.

Atomiclike states in $\mathrm{La}_{2} \mathrm{CuO}_{4}$ require an orthorhombic distortion of the tetragonal crystal. Hence, the total condensation energy $\Delta E_{i}(x)$ decreases by the energy $\Delta E_{i}^{d}(x)$ required to distort the tetragonal crystal. The $x$-dependence of this "distortion energy" might be understood on the basis of the following assumptions.

With increasing $x$ the translation symmetry of the crystal is increasingly disturbed and the points $\Gamma$ and $X$ in the tetragonal Brillouin zone become increasingly equivalent. As a consequence, the orthorhombic distortion that is required for that $\Gamma$ and $X$ are (completely) equivalent, becomes smaller with increasing $x$ (and disappears

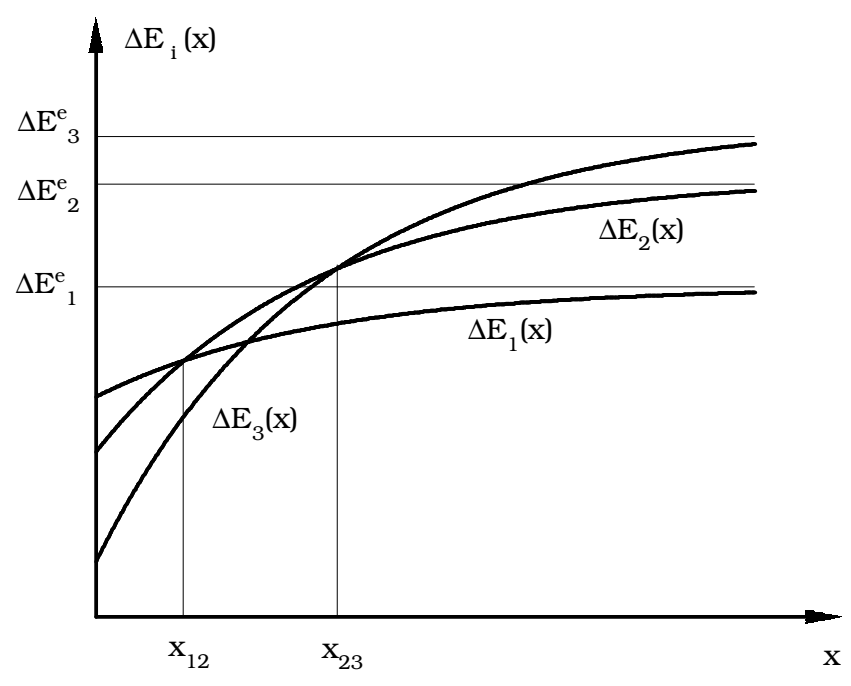

FIG. 9: Qualitative plot of the condensation energy $\Delta E_{i}(x)$ [given in Eq. (11)] as a function of the Sr concentration $x$ within the simple model defined by the three assumptions (12), (13), and (14). $i=1,2$, and 3 labels the antiferromagnetic, the neutral, and the spin-boson phase, respectively. The condensation energy is maximum for any concentration, if the phase transitions from the antiferromagnetic to the neutral and from the neutral to the spin-boson phase occur at the Sr concentrations $x_{12}$ and $x_{23}$, respectively. Below a transition temperature, the spin-boson phase is superconducting.

completely for $x \approx 0.2$ ). Thus, for fixed $i$, the required distortion energy $\Delta E_{i}^{d}(x)$ decreases with increasing $x$ :

$$
\Delta E_{i}^{d}\left(x_{1}\right)>\Delta E_{i}^{d}\left(x_{2}\right) \quad \text { if } \quad x_{1}<x_{2} .
$$

On the basis of this assumption, the experimental results may be interpreted by assuming that, first, the purely electronic condensation energy is maximum for the spin-boson and minimum for the antiferromagnetic state,

$$
\Delta E_{1}^{e}<\Delta E_{2}^{e}<\Delta E_{3}^{e},
$$

and that, second, for fixed $x$, the required distortion energy is also maximum for the spin-boson and minimum for the antiferromagnetic state,

$$
\Delta E_{1}^{d}(x)<\Delta E_{2}^{d}(x)<\Delta E_{3}^{d}(x) .
$$

Under these assumptions, the system undergoes the phase transitions in order that the condensation energy is maximum at any $\mathrm{Sr}$ concentration $x$, see Fig. 9.

The first assumption (13) is in accordance with my observations on the elemental metals, see Sec. V of Ref. [4]. The second assumption is corroborated by the fact that a spin structure alone (together with the related magnetostriction) represents a distortion of the lattice without any spatial distortion. Hence, it is conceivable that the required spatial distortion of the tetragonal crystal is smallest in the antiferromagnetic state. 


\section{APPENDIX A: STABLE MAGNETIC STATES}

Consider a magnetic state $|m\rangle$ and the related magnetic group

$$
M=H+A H,
$$

where $H$ denotes the space group and $A$ stands for any anti-unitary element of $M$. In the case of the spin structure depicted in Fig. 6, we have $H=C m c a$ and may choose $A=\left\{K \mid \frac{1}{2} \frac{1}{2} \frac{1}{2}\right\}$ with $K$ denoting the operator of time-inversion.

Within the NHM, both the magnetic state $|m\rangle$ and the time-inverted state

$$
\overline{|m\rangle}=K|m\rangle
$$

are eigenstates of the nonadiabatic Hamiltonian $H^{n}$. Therefore, standard group-theoretical methods can be applied to magnetic states, too. A stable magnetic state $|m\rangle$ complies with two conditions:

- $|m\rangle$ is basis function of a one-dimensional corepresentation of $M$;

- $|m\rangle$ and the time-inverted state $\overline{|m\rangle}$ are basis functions of a two-dimensional irreducible corepresentation of the gray magnetic group

$$
\bar{M}=M+K M,
$$

see Sec. III.C of Ref. [5].

Consequently, a stable magnetic state can exist in the space group $H$ if $H$ has at least one one-dimensional single-valued representation $R$ following

- case (a) with respect to the magnetic group $H+A H$ and

- case (c) with respect to the magnetic group $H+K H$.

The cases (a) and (c) are defined in Eqs. (7.3.45) and (7.3.47), respectively, in the textbook of Bradley and Cracknell [16]. The irreducible corepresentation derived from $R$ stays one-dimensional in case (a) and becomes two-dimensional in case (c).

\section{Space group $\mathrm{Cmca}$}

Table II lists all the irreducible representations of the space group $\mathrm{H}=\mathrm{Cmca}$ of orthorhombic $\mathrm{La}_{2} \mathrm{CuO}_{4}$. The second and third column of this Table specify whether the corepresentation of $H+K H$ and $H+\left\{K \mid \frac{1}{2} \frac{1}{2} \frac{1}{2}\right\} H$, respectively, derived from the given representation $R$ follows case (a) or case (c).

The only one-dimensional representations which apparently comply with the above condition for a stable antiferromagnetic state are the small representations at point $R$. However, the little group at $R$ is not the complete space group. Therefore, a stable antiferromagnetic state with the space group $C m c a$ does not exist.

\section{Space group Pccn}

Table III lists all the irreducible representations of the space group $H=P c c n$. The second, third, and fourth column of this table specify whether the corepresentation of $H+K H, H+\left\{K \mid \frac{1}{2} \frac{1}{2} 0\right\} H$, and $H+\left\{K \mid \frac{1}{2} 0 \frac{1}{2}\right\} H$ [cf. Eqs. (8) and (9)], respectively, derived from the given representation $R$ follows case (a) or case (c).

For the group $H+\left\{K \mid \frac{1}{2} 0 \frac{1}{2}\right\} H$ there do not exist suitable representations. However, for the group $H+$ $\left\{K \mid \frac{1}{2} \frac{1}{2} 0\right\} H$ all the small representations at point $R$ comply with the above condition for a stable antiferromagnetic state. Since now the little group at $R$ includes all the space-group elements, a stable antiferromagnetic state with the space group Pccn is possible.

\section{APPENDIX B: OPTIMALLY LOCALIZABLE AND SYMMETRY-ADAPTED WANNIER FUNCTIONS}

\section{Usual (spin-independent) Wannier functions}

Consider a subgroup $H$ of the space group $I 4 / \mathrm{mmm}$ of $\mathrm{La}_{2} \mathrm{CuO}_{4}$ and assume the energy bands (or a part of the energy bands) of $\mathrm{La}_{2} \mathrm{CuO}_{4}$ to be folded into the Brillouin zone $B_{H}$ of $H$. Let be $\mu$ the number of $\mathrm{Cu}$ atoms in the unit cell of $H$ and consider $\mu$ single energy bands in $B_{H}$. Assume the Bloch functions $\varphi_{\vec{k} q}(\vec{r})$ (with the wave vector $\vec{k}$ ) of this "energy band with $\mu$ branches" to be unitarily transformed into Wannier functions

$$
w_{i}\left(\vec{r}-\vec{T}-\vec{\rho}_{i}\right)=\frac{1}{\sqrt{\nu}} \sum_{\vec{k}}^{B Z} \sum_{q=1}^{\mu} e^{-i \vec{k}\left(\vec{T}+\vec{\rho}_{i}\right)} g_{i q}(\vec{k}) \varphi_{\vec{k} q}(\vec{r})
$$

centered at the $\mathrm{Cu}$ atoms. The first sum in this equation runs over the $\nu$ vectors $\vec{k}$ of the first Brillouin zone (BZ), the second sum runs over the $\mu$ branches, $\vec{T}$ and $\vec{\rho}_{i}$ denote the vectors of the Bravais lattice and the positions of the $\mathrm{Cu}$ atoms within the unit cell, respectively, and the coefficients $g_{i q}(\vec{k})$ are the elements of a unitary matrix $\mathbf{g}(\vec{k})$,

$$
\mathbf{g}^{-1}(\vec{k})=\mathbf{g}^{\dagger}(\vec{k})
$$

The Wannier functions are called "optimally localizable" if the Bloch-like functions

$$
\widetilde{\varphi}_{\vec{k} i}(\vec{r})=\sum_{q=1}^{\mu} g_{i q}(\vec{k}) \varphi_{\vec{k} q}(\vec{r})
$$

are (for fixed $\vec{r}$ ) continuous functions of $\vec{k}$ everywhere in the reciprocal space. Fortunately, the question whether or not the functions $\widetilde{\varphi}_{\vec{k} i}(\vec{r})$ vary continuously through the planes of symmetry within or on the surface of the Brillouin zone, can be treated by group theory [17, 18]. 
The main result is the relatively simple criterion given in Eq. (B7).

The Wannier functions are called "symmetry-adapted to $H$ " if they satisfy the equation

$$
P(a) w_{i}\left(\vec{r}-\vec{T}-\vec{\rho}_{i}\right)=\sum_{j=1}^{\mu} D_{j i}(\alpha) w_{j}\left(\vec{r}-\alpha \vec{T}-\vec{\rho}_{j}\right)
$$

by application of the operators $P(a)$ of $H$,

$$
a=\{\alpha \mid \vec{t}\} \in H,
$$

see Appendix A of Ref. [6]. The matrices $\mathbf{D}_{0}(\alpha)=$ $\left[D_{j i}(\alpha)\right]$ form a (reducible or irreducible) single-valued representation $D_{0}$ of the point group $H_{0}$ of $H$. The sum on the right hand side of Eq. (B4) consists of one summand only because there is one Wannier function at each atom. Hence, the matrices $\mathbf{D}_{0}(\alpha)$ have only one non-vanishing element, say $d_{j i}(\alpha)$, in each row and each column. The non-vanishing diagonal elements $d_{j j}(\alpha)$ are equal to $\chi_{0}(\alpha)$ and the other non-vanishing elements may also be chosen to be equal to $\chi_{0}(\alpha)$, so that we have

$$
d_{j i}(\alpha)=\chi_{0}(\alpha)
$$

for all the non-vanishing elements of $\mathbf{D}_{0}(\alpha)$, where $\chi_{0}(\alpha)$ stands for the character of the one-dimensional representation $d_{0}$ of $H_{0}$ used in the following Eq. (B7).

Suppose the symmetry of the Bloch functions in the Brillouin zone $B_{H}$ of $H$ to be determined by the compatibility relations given in the Tables VI - VIII. At each point of symmetry $P_{\vec{k}}$ of $B_{H}$ the Bloch functions of the $\mu$ branches of the band belong to a $\mu$-dimensional singlevalued small representation $D_{\vec{k}}$ of the little group $H_{\vec{k}}$ of $P_{\vec{k}}$. $D_{\vec{k}}$ may be irreducible or is the direct sum over small irreducible representations of $H_{\vec{k}}$.

The coefficients $g_{i q}(\vec{k})$ can be chosen so that the Wannier functions in Eq. (B1) are optimally localizable and symmetry-adapted to $H$ if and only if the equation

$$
\chi_{\vec{k}}(a)=\chi_{0}(\alpha) e^{-i \alpha \vec{k} \cdot \vec{t}} \sum_{i=1}^{\mu} n_{i}(a) e^{-i \overrightarrow{\varrho_{i}} \cdot(\vec{k}-\alpha \vec{k})}
$$

is satisfied at each $P_{\vec{k}}$ of $B_{H}$ and for each element $a=\{\alpha \mid \vec{t}\}$ in the little group $H_{\vec{k}} \cdot \chi_{\vec{k}}(a)$ denotes the character of the representation $D_{\vec{k}}, \chi_{0}(\alpha)$ stands for the character of any one-dimensional single-valued representation $d_{0}$ of the point group $H_{0}$ of $H$, and

$$
n_{i}(a)= \begin{cases}1 & \text { if } \alpha \vec{\varrho}_{i}+\vec{t}=\vec{\varrho}_{i}+\vec{T} \\ 0 & \text { else }\end{cases}
$$

with $\vec{T}$ being a vector of the Bravais lattice. This Eq. (B7) is derived from Eqs. (1.8) and (4.28) of Ref. [17] (see also Sec. 3 of Ref. [18]). It is satisfied for all $a \in H_{\vec{k}}$ if it is satisfied for one element of each class of $H_{\vec{k}}$.

\section{Spin-dependent Wannier functions}

The Wannier functions given in Eq. (B1) become spindependent if the Bloch functions $\varphi_{\vec{k} q}(\vec{r})$ in this equation are allowed to have $\vec{k}$-dependent spin-directions, see Eqs. (A21) and (A22) in Ref. [6]. Also energy bands with optimally localizable and symmetry-adapted spindependent Wannier functions situated at the $\mathrm{Cu}$ atoms follow Eq. (B7). In this case, however, $D_{\vec{k}}$ is a $2 \mu$ dimensional double-valued representation and $\chi_{0}(\alpha)$ denotes the character of any two-dimensional double-valued representation $d_{0}$ of $H_{0}$.

\section{Magnetic groups}

The Wannier functions given in Eq. (B1) are called symmetry-adapted to the antiferromagnetic group

$$
M=H+\{K \mid \vec{\tau}\} H
$$

if the equation

$$
K P(\{E \mid \vec{\tau}\}) w_{i}\left(\vec{r}-\vec{T}-\vec{\rho}_{i}\right)=\sum_{j=1}^{\mu} N_{j i} w_{j}\left(\vec{r}-\vec{T}-\vec{\rho}_{j}\right)
$$

is satisfied in addition to Eq. (B4), where $K$ still denotes the operator of time-inversion. Since there is one Wannier function at each atom, also the matrix $\mathbf{N}=\left[N_{j i}\right]$ has only one non-vanishing element, say $n_{j i}$, in each row and each column which may be put equal to one,

$$
n_{j i}=1 .
$$

Suppose that in Eq. (B10) we obtain the Wannier function $w_{j}$ by application of the operator $K P(\{E \mid \vec{\tau}\})$ on the function $w_{i}$. Then conversely we obtain the function $w_{i}$ applying this operator on $w_{j}$. This is because $2 \tau$ is a vector of the Bravais lattice,

$$
2 \vec{\tau}=\vec{T},
$$

and hence from $\vec{\rho}_{i}+\vec{\tau}=\vec{\rho}_{j}+\vec{T}_{1}$ it follows $\vec{\rho}_{j}+\vec{\tau}=\vec{\rho}_{i}+\vec{T}_{2}$, where $\vec{T}_{1}$ and $\vec{T}_{2}$ denote vectors of the Bravais lattice. Thus, the matrix $\mathbf{N}$ is symmetric,

$$
n_{j i}=n_{i j} .
$$

$\mathbf{N}$ is the matrix representing $K$ in the corepresentation of the point group

$$
M_{0}=H_{0}+K H_{0}
$$

of $M$ which is derived from the representation $D_{0}$ of $H_{0}$ given in Eq. (B4). Hence, $\mathbf{N}$ must satisfy the equations

$$
\mathrm{NN}^{*}=1
$$

and

$$
\mathbf{D}_{0}(\alpha)=\mathbf{N D}_{0}^{*}(\alpha) \mathbf{N}^{-1} \text { for } \alpha \in H_{0},
$$


see Eq. (7.3.45) of Ref. [16]. The matrices $\mathbf{D}_{0}(\alpha)$ are defined in Eq. (B4). [In Ref. [16] Eq. (7.3.45) is derived for irreducible representations. However, this derivation in Sec. 7.3 of Ref. [16] shows that Eq. (7.3.45) holds for reducible representations, too, if Eq. (B15) is satisfied.]

As a consequence of Eqs. (B11) and (B13), the first Eq. (B15) is satisfied (and hence $\mathbf{N}=\mathbf{N}^{*}=\mathbf{N}^{-1}$ ). The validity of the second Eq. (B16) must be examined for each given magnetic group.

Assume Eq. (B7) to be valid in a given energy band. In Ref. [19] I have shown that then the coefficients $g_{i q}(\vec{k})$ [in Eq. (B1)] can be chosen so that the Wannier functions of this band are symmetry-adapted to the whole magnetic group $M$ if the matrix $\mathbf{N}$ satisfies Eq. (B16) and the equation

$$
\mathbf{S}^{*}(-\vec{K})=\mathbf{N S}(\vec{K}) \mathbf{N}^{-1} \cdot e^{i \vec{K} \cdot \tau}
$$

for any vector $\vec{K}$ of the reciprocal lattice, where $\mathbf{S}(\vec{K})$ is a $\mu$-dimensional diagonal matrix with the elements

$$
s_{j j}=e^{i \vec{K} \cdot \vec{\rho}_{j}}
$$

Using Eqs. (B11), (B12), and (B13) it can be verified that the matrix $\mathbf{N}$ defined by Eq. (B10) satisfies Eq. (B17). Thus, Eq. (B16) is the only additional condition for the existence of optimally localizable Wannier functions that are symmetry-adapted to the entire magnetic group $M$. This equation is satisfied for all the antiferromagnetic bands considered in this paper.

\section{APPENDIX C: DESCRIPTION OF THE SPACE GROUPS}

\section{Tetragonal $\mathrm{La}_{2} \mathrm{CuO}_{4}$}

Fig. 1 shows the basic translations of the space group $I 4 / m m m$ of tetragonal $\mathrm{La}_{2} \mathrm{CuO}_{4}$ and the Brillouin zone is drawn in Fig. 4 (top).

The point group $D_{4 h}$ consists of 16 elements,

$$
\begin{aligned}
D_{4 h}= & \left\{E, C_{4 z}^{+}, C_{4 z}^{-}, C_{2 z}, C_{2 x}, C_{2 y}, C_{2 a}, C_{2 b}, I\right. \\
& S_{4 z}^{-}=I C_{4 z}^{+}, S_{4 z}^{+}=I C_{4 z}^{-}, \sigma_{z}=I C_{2 z}, \sigma_{x}=I C_{2 x} \\
& \left.\sigma_{y}=I C_{2 y}, \sigma_{d a}=I C_{2 a}, \sigma_{d b}=I C_{2 b}\right\}
\end{aligned}
$$

representing the identity operation, anti-clockwise and clockwise rotation through $\pi / 2$ radians about the $z$ axis, rotation through $\pi$ radians about the $z, x$ and $y$ axes, rotation through $\pi$ radians about the $a$ and $b$ axes drawn in Fig. 1, and inversion. The following elements are products of the inversion with the former elements.

The space group $I 4 / \mathrm{mmm}$ is symmorphic. Hence, all the point group elements are elements of $14 / \mathrm{mmm}$, too.

\section{Orthorhombic $\mathrm{La}_{2} \mathrm{CuO}_{4}$}

The space group $C m c a$ of orthorhombic $\mathrm{La}_{2} \mathrm{CuO}_{4}$ has the basic translations shown in Fig. 2. The basic domain of the Brillouin zone of Cmca is drawn in Fig. 4 (center).

Within the coordinate system given in Fig. 2 its point group is

$$
D_{2 h}=\left\{E, C_{2 z}, C_{2 a}, C_{2 b}, I, \sigma_{z}, \sigma_{d a}, \sigma_{d b}\right\} .
$$

In the space group Cmca the 4 elements

$$
C_{2 z}, C_{2 a}, \sigma_{z}, \text { and } \sigma_{d a}
$$

are associated with the nonprimitive translation

$$
\left(\frac{1}{2} \frac{1}{2} \frac{1}{2}\right)
$$

\section{Antiferromagnetic $\mathrm{La}_{2} \mathrm{CuO}_{4}$}

Fig. 7 shows the spin structure of $\mathrm{La}_{2} \mathrm{CuO}_{4}$ as determined by Keimer et al. [1]. The space group is Pccn with the Bravais lattice $\Gamma_{o}$. Fig. 8 gives the basic translations as used in this paper, and the basic domain of the Brillouin zone is drawn in Fig. 4 (bottom).

The point group of the antiferromagnetic structure is also the group $D_{2 h}$ given in Eq. (C2). In the space group Pccn the 2 elements

$$
C_{2 z} \text { and } \sigma_{z}
$$

are associated with the nonprimitive translation

$$
\left(\frac{1}{2} \frac{1}{2} 0\right)
$$

the elements

$$
C_{2 a} \text { and } \sigma_{d a}
$$

are associated with

$$
\left(0 \frac{1}{2} \frac{1}{2}\right)
$$

and the elements

$$
C_{2 b} \text { and } \sigma_{d b}
$$

are associated with

$$
\left(\frac{1}{2} 0 \frac{1}{2}\right)
$$

\section{APPENDIX D: TABLES}

\section{Irreducible representations}

The irreducible representations given in Tables I - V (on the following pages) are determined by means of Tables 5.7 and 6.13 in the textbook of Bradley and Cracknell [16], with the notations of tables 5.8 and 6.14 in this book. The cases (a), (b), and (c) of the corepresentations follow Eqs. (7.3.45), (7.3.46), and (7.3.47) and are determined by Eq. (7.3.51) of Ref. [16]. 


\section{Compatibility tables}

Tables VI - VIII list compatibility relations between the points of symmetry in the Brillouin zone of a space group $G$ and the related points of symmetry in the Brillouin zone of a subgroup $H$ of $G$. These tables are determined in the way described in great detail in Ref. [15]. The compatibility relations between single-valued and double-valued representations given in Tables IX and X are determined by standard group-theoretical methods.

\section{Antiferromagnetic, neutral, and superconducting bands}

Tables XI, XIV, XV, and XVI list all the single-valued representations $D_{\vec{k}}$ with characters $\chi_{\vec{k}}(a)$ satisfying Eq. (B7) in the space groups $H=$
$I 4 / m m m, C m c a, P 4 / m n c$, and Pccn, respectively. Different bands belong to the character $\chi_{0}(\alpha)$ of different one-dimensional representations $d_{0}$ of $H_{0}$. Tables XII and XIII list all the double-valued representations satisfying this equation with $H=I 4 / \mathrm{mmm}$ and $\mathrm{Cmca}$, respectively. In this case, however, $D_{\vec{k}}$ is a $2 \mu$-dimensional double-valued representation and $\chi_{0}(\alpha)$ denotes the character of any two-dimensional double-valued representation $d_{0}$ of $H_{0}$.

\section{ACKNOWLEDGMENTS}

I am very indebted to Ove Jepsen for providing me with all the data I needed to determine the symmetry of the Bloch functions in the band structure of $\mathrm{La}_{2} \mathrm{CuO}_{4}$. I thank Ernst Helmut Brandt for critical comments on the manuscript and continuing encouragement.
1. B. Keimer, N. Belk, R. J. Birgeneau, A. Cassanho, C. Y. Chen, M. Greven, M. A. Kastner, A. Aharony, Y. Endoh, R. W. Erwin, and G. Shirane, Phys. Rev. B 46, 14034 (1992).

2. R. J. Cava, A. Santoro, D. W. J. Jr., and W. W. Rhodes, Phys. Rev. B 35, 6716 (1987).

3. B. J. Sternlieb, G. M. Luke, Y. J. Uemura, T. M. Riseman, J. H. Brewer, P. M. Gehring, K. Yamada, Y. Hidaka, T. Murakami, T. R. Thurston, and R. J. Birgeneau, Phys. Rev. B 41, 8866 (1990).

4. E. Krüger, Phys. Rev. B 59, 13795 (1999).

5. E. Krüger, Phys. Rev. B 40, 11090 (1989).

6. E. Krüger, Phys. Rev. B 63, 144403 (2001).

7. E. Krüger, J. Supercond. 14(4), 469 (2001).

8. E. Krüger, J. Supercond. 14(4), 551 (2001).

9. E. Krüger, J. Supercond. 15(2), 105 (2002).
10. N. F. Mott, Can. J. Phys. 34, 1356 (1956).

11. J. Hubbard, Proc. R. Soc. London, Ser. A 276, 238 (1963).

12. J. Bardeen, L. N. Cooper, and J. R. Schrieffer, Phys. Rev. 108, 1175 (1957).

13. E. Krüger, phys. stat. sol. b 156, 345 (1989).

14. O. Jepsen, private communication.

15. E. Krüger, Phys. Rev. B 32, 7493 (1985).

16. C. Bradley and A.P.Cracknell, The Mathematical Theory of Symmetry in Solids (Claredon, Oxford, 1972).

17. E. Krüger, phys. stat. sol. b 52, 215 (1972).

18. E. Krüger, phys. stat. sol. b 52, 519 (1972).

19. E. Krüger, phys. stat. sol. b 61, 193 (1974).

20. E. Krüger, Phys. Rev. B 36, 2263 (1987). 
TABLE I: Characters of the small (allowed) single-valued irreducible representations of the space group $I 4 / m m m=D_{4 h}^{17}(139)$ of tetragonal $\mathrm{La}_{2} \mathrm{CuO}_{4}$ in the notation of Table 5.8 in the textbook of Bradley and Cracknell [16].

$$
\begin{array}{clll}
\Gamma(000) & \text { and } Z\left(\frac{1}{2} \frac{1}{2} \frac{\overline{1}}{2}\right) \\
C_{4 z}^{-} & C_{2 y} & C_{2 b} & \\
C_{4 z}^{+} & \sigma_{y} & \sigma_{d b}
\end{array}
$$

\begin{tabular}{cccccccccc}
$E$ & $C_{2 z}$ & $C_{4 z}^{+}$ & $C_{2 x}$ & $C_{2 a}$ & $I$ & $\sigma_{z}$ & $S_{4 z}^{-}$ & $\sigma_{x}$ & $\sigma_{d a}$ \\
\hline
\end{tabular}

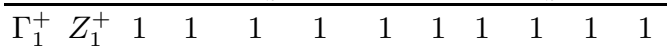

$\begin{array}{llllllllllll}\Gamma_{2}^{+} & Z_{2}^{+} & 1 & 1 & 1 & -1 & -1 & 1 & 1 & 1 & -1 & -1\end{array}$

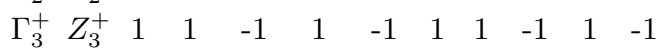

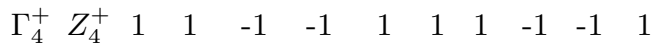

$\begin{array}{llllllllllll}\Gamma_{5}^{+} & Z_{5}^{+} & 2 & -2 & 0 & 0 & 0 & 2 & -2 & 0 & 0 & 0\end{array}$

$\begin{array}{llllllllllll}\Gamma_{1}^{-} & Z_{1}^{-} & 1 & 1 & 1 & 1 & 1 & -1 & -1 & -1 & -1 & -1\end{array}$

$\begin{array}{llllllllllll}\Gamma_{2}^{-} & Z_{2}^{-} & 1 & 1 & 1 & -1 & -1 & -1 & -1 & -1 & 1 & 1\end{array}$

$\begin{array}{llllllllllll}\Gamma_{3}^{-} & Z_{3}^{-} & 1 & 1 & -1 & 1 & -1 & -1 & -1 & 1 & -1 & 1\end{array}$

$\begin{array}{llllllllllll}\Gamma_{4}^{-} & Z_{4}^{-} & 1 & 1 & -1 & -1 & 1 & -1 & -1 & 1 & 1 & -1\end{array}$

\begin{tabular}{|c|c|c|c|c|c|c|c|c|}
\hline & & & & & $I^{\prime}$ & $\sigma_{y}$ & & \\
\hline & & $\overline{N_{1}^{+}}$ & 1 & 1 & 1 & 1 & & \\
\hline & & $N_{1}^{-}$ & 1 & 1 & -1 & -1 & & \\
\hline & & $N_{2}^{+}$ & 1 & -1 & 1 & -1 & & \\
\hline & & $\mathrm{N}_{2}^{-}$ & 1 & -1 & -1 & 1 & & \\
\hline & & & $X$ & $\left(00 \frac{1}{2}\right.$ & & & & \\
\hline & $E$ & $C_{2 z}$ & $C_{2 a}$ & $C_{2 b}$ & $I$ & $\sigma_{z}$ & $\sigma_{d a}$ & $\sigma_{d b}$ \\
\hline$\overline{X_{1}^{+}}$ & 1 & 1 & 1 & 1 & 1 & 1 & 1 & 1 \\
\hline$X_{2}^{+}$ & 1 & -1 & 1 & -1 & 1 & -1 & 1 & -1 \\
\hline$X_{3}^{+}$ & 1 & 1 & -1 & -1 & 1 & 1 & -1 & -1 \\
\hline$X_{4}^{+}$ & 1 & -1 & -1 & 1 & 1 & -1 & -1 & 1 \\
\hline$X_{1}^{-}$ & 1 & 1 & 1 & 1 & -1 & -1 & -1 & -1 \\
\hline$X_{2}^{-}$ & 1 & -1 & 1 & -1 & -1 & 1 & -1 & 1 \\
\hline$X_{3}^{-}$ & 1 & 1 & -1 & -1 & -1 & -1 & 1 & 1 \\
\hline$X_{4}^{-}$ & 1 & -1 & -1 & 1 & -1 & 1 & 1 & -1 \\
\hline
\end{tabular}

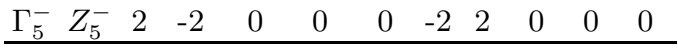

\begin{tabular}{|c|c|c|c|c|}
\hline & & $\begin{array}{l}\frac{1}{4} \frac{1}{4} \frac{1}{4} \\
S_{4 z}^{-} \\
S_{4 z}^{+}\end{array}$ & $\begin{array}{l}C_{2 y} \\
C_{2 x}\end{array}$ & $\begin{array}{l}\sigma_{d a} \\
\sigma_{d b}\end{array}$ \\
\hline$\overline{P_{1} 1}$ & $\begin{array}{ll}1 \\
\end{array}$ & 1 & 1 & 1 \\
\hline$P_{2}$ & $\begin{array}{ll}1 & 1\end{array}$ & 1 & -1 & -1 \\
\hline$P_{3} 1$ & $\begin{array}{ll}1 & 1\end{array}$ & -1 & 1 & -1 \\
\hline$P_{4} 1$ & $1 \quad 1$ & -1 & -1 & 1 \\
\hline$P_{5} 2$ & $\begin{array}{ll}2 & -2\end{array}$ & 0 & 0 & 0 \\
\hline
\end{tabular}


TABLE II: Characters of the small (allowed) single-valued irreducible representations of the space group $H=C m c a=D_{2 h}^{18}(64)$ of the orthorhombic phase of $\mathrm{La}_{2} \mathrm{CuO}_{4}$ in the notation of Table 5.8 in the textbook of Bradley and Cracknell [16]. $K$ stands for the operator of time-inversion. The first two elements in the first row define two magnetic groups $M_{1}=H+K H \quad$ and $\quad M_{2}=$ $H+\left\{K \mid \frac{1}{2} \frac{1}{2} \frac{1}{2}\right\} H$. The second and third column of the table specify whether the corepresentations of $M_{1}$ and $M_{2}$, respectively, derived from the given representations $R_{i}$ of $H$ follow case $(a)$, case $(b)$, or case $(c)$ when they are given by Eqs. (7.3.45-47) of Ref. [16]. In case (a), there is no change in the degeneracy of $R_{i}$, and in case (c) the degeneracy becomes doubled.

$$
\Gamma(000) \text { and } Y\left(\frac{1}{2} \frac{1}{2} 0\right)
$$

$K\left\{K \mid \frac{1}{2} \frac{1}{2} \frac{1}{2}\right\}\{E \mid 000\}\left\{C_{2 a} \mid \frac{1}{2} \frac{1}{2} \frac{1}{2}\right\}\left\{C_{2 z} \mid \frac{1}{2} \frac{1}{2} \frac{1}{2}\right\}\left\{C_{2 b} \mid 000\right\}\{I \mid 000\} \quad\left\{\sigma_{d a} \mid \frac{1}{2} \frac{1}{2} \frac{1}{2}\right\}\left\{\sigma_{z} \mid \frac{1}{2} \frac{1}{2} \frac{1}{2}\right\}\left\{\sigma_{d b} \mid 000\right\}$

\begin{tabular}{|c|c|c|c|c|c|c|c|c|c|}
\hline$\overline{\Gamma_{1}^{+}} Y_{1}^{+}$(a) & (a) & 1 & 1 & 1 & 1 & 1 & 1 & 1 & 1 \\
\hline$\Gamma_{2}^{+} Y_{2}^{+}(\mathrm{a})$ & (a) & 1 & -1 & 1 & -1 & 1 & -1 & 1 & -1 \\
\hline$\Gamma_{3}^{+} Y_{3}^{+}(\mathrm{a})$ & (a) & 1 & 1 & -1 & -1 & 1 & 1 & -1 & -1 \\
\hline$\Gamma_{4}^{+} Y_{4}^{+}(\mathrm{a})$ & (a) & 1 & -1 & -1 & 1 & 1 & -1 & -1 & 1 \\
\hline$\Gamma_{1}^{-} Y_{1}^{-}$(a) & (a) & 1 & 1 & 1 & 1 & -1 & -1 & -1 & -1 \\
\hline$\Gamma_{2}^{-} Y_{2}^{-}(\mathrm{a})$ & (a) & 1 & -1 & 1 & -1 & -1 & 1 & -1 & 1 \\
\hline$\Gamma_{3}^{-} Y_{3}^{-}(\mathrm{a})$ & (a) & 1 & 1 & -1 & -1 & -1 & -1 & 1 & 1 \\
\hline$\Gamma_{4}^{-} Y_{4}^{-}$(a) & (a) & 1 & -1 & -1 & 1 & -1 & 1 & 1 & -1 \\
\hline
\end{tabular}

$$
\begin{gathered}
Z\left(00 \frac{1}{2}\right) \text { and } T\left(\frac{1}{2} \frac{1}{2} \frac{1}{2}\right) \\
\left\{C_{2 a} \mid \frac{1}{2} \frac{1}{2} \frac{3}{2}\right\}\{I \mid 001\}\left\{\sigma_{d a} \mid \frac{1}{2} \frac{1}{2} \frac{3}{2}\right\}
\end{gathered}
$$$$
\left\{\sigma_{z} \mid \frac{1}{2} \frac{1}{2} \frac{3}{2}\right\}\left\{C_{2 b} \mid 001\right\}\left\{C_{2 z} \mid \frac{1}{2} \frac{1}{2} \frac{3}{2}\right\}
$$

$K\left\{K \mid \frac{1}{2} \frac{1}{2} \frac{1}{2}\right\}\{E \mid 000\}\{E \mid 001\}\left\{C_{2 a} \mid \frac{1}{2} \frac{1}{2} \frac{1}{2}\right\}\{I \mid 000\}\left\{\sigma_{d a} \mid \frac{1}{2} \frac{1}{2} \frac{1}{2}\right\}\left\{\sigma_{d b} \mid 000\right\}\left\{\sigma_{d b} \mid 001\right\}\left\{\sigma_{z} \mid \frac{1}{2} \frac{1}{2} \frac{1}{2}\right\}\left\{C_{2 b} \mid 000\right\} \quad\left\{C_{2 z} \mid \frac{1}{2} \frac{1}{2} \frac{1}{2}\right\}$

\begin{tabular}{llllllllllll}
\hline$Z_{1}$ (a) & (a) & 2 & -2 & 0 & 0 & 0 & 2 & -2 & 0 & 0 & 0 \\
$Z_{2}$ (a) & (a) & 2 & -2 & 0 & 0 & 0 & -2 & 2 & 0 & 0 & 0 \\
\hline
\end{tabular}

\begin{tabular}{ccccccccccc}
\multicolumn{10}{c}{$S\left(0 \frac{1}{2} 0\right)$} \\
\end{tabular}


TABLE III: Characters of the small (allowed) single-valued irreducible representations of the space group $H=P c c n=D_{2 h}^{10}$ (56) of the antiferromagnetic structure of $\mathrm{La}_{2} \mathrm{CuO}_{4}$ in the notation of Table 5.8 in the textbook of Bradley and Cracknell [16]. $K$ stands for the operator of time-inversion. The first three elements in the first row define three magnetic groups $M_{1}=H+K H, M_{2}=H+\left\{K \mid \frac{1}{2} \frac{1}{2} 0\right\} H$, and $M_{3}=H+\left\{K \mid \frac{1}{2} 0 \frac{1}{2}\right\} H$. The second, third, and fourth column of the table specify whether the corepresentations of $M_{1}, M_{2}$, and $M_{3}$, respectively, derived from the given representations $R_{i}$ of $H$ follow case ( $a$ ), case $(b)$, or case $(c)$ when they are given by Eqs. (7.3.45-47) of Ref. [16]. In case (a), there is no change in the degeneracy of $R_{i}$, and in case (c) the degeneracy becomes doubled.

$\Gamma(000)$

\begin{tabular}{|c|c|c|c|c|c|c|c|c|c|c|}
\hline$\overline{\Gamma_{1}^{+}(\mathrm{a})}$ & (a) & (a) & 1 & 1 & 1 & 1 & 1 & 1 & 1 & 1 \\
\hline$\Gamma_{2}^{+}(\mathrm{a})$ & (a) & (a) & 1 & -1 & 1 & -1 & 1 & -1 & 1 & -1 \\
\hline$\Gamma_{3}^{+} \quad(\mathrm{a})$ & (a) & (a) & 1 & 1 & -1 & -1 & 1 & 1 & -1 & -1 \\
\hline$\Gamma_{4}^{+}(\mathrm{a})$ & (a) & (a) & 1 & -1 & -1 & 1 & 1 & -1 & -1 & 1 \\
\hline$\Gamma_{1}^{-}(\mathrm{a})$ & (a) & (a) & 1 & 1 & 1 & 1 & -1 & -1 & -1 & -1 \\
\hline$\Gamma_{2}^{-} \quad(\mathrm{a})$ & (a) & (a) & 1 & -1 & 1 & -1 & -1 & 1 & -1 & 1 \\
\hline$\Gamma_{3}^{-}(\mathrm{a})$ & (a) & (a) & 1 & 1 & -1 & -1 & -1 & -1 & 1 & 1 \\
\hline$\Gamma_{4}^{-}(\mathrm{a})$ & (a) & (a) & 1 & -1 & -1 & 1 & -1 & 1 & 1 & -1 \\
\hline
\end{tabular}

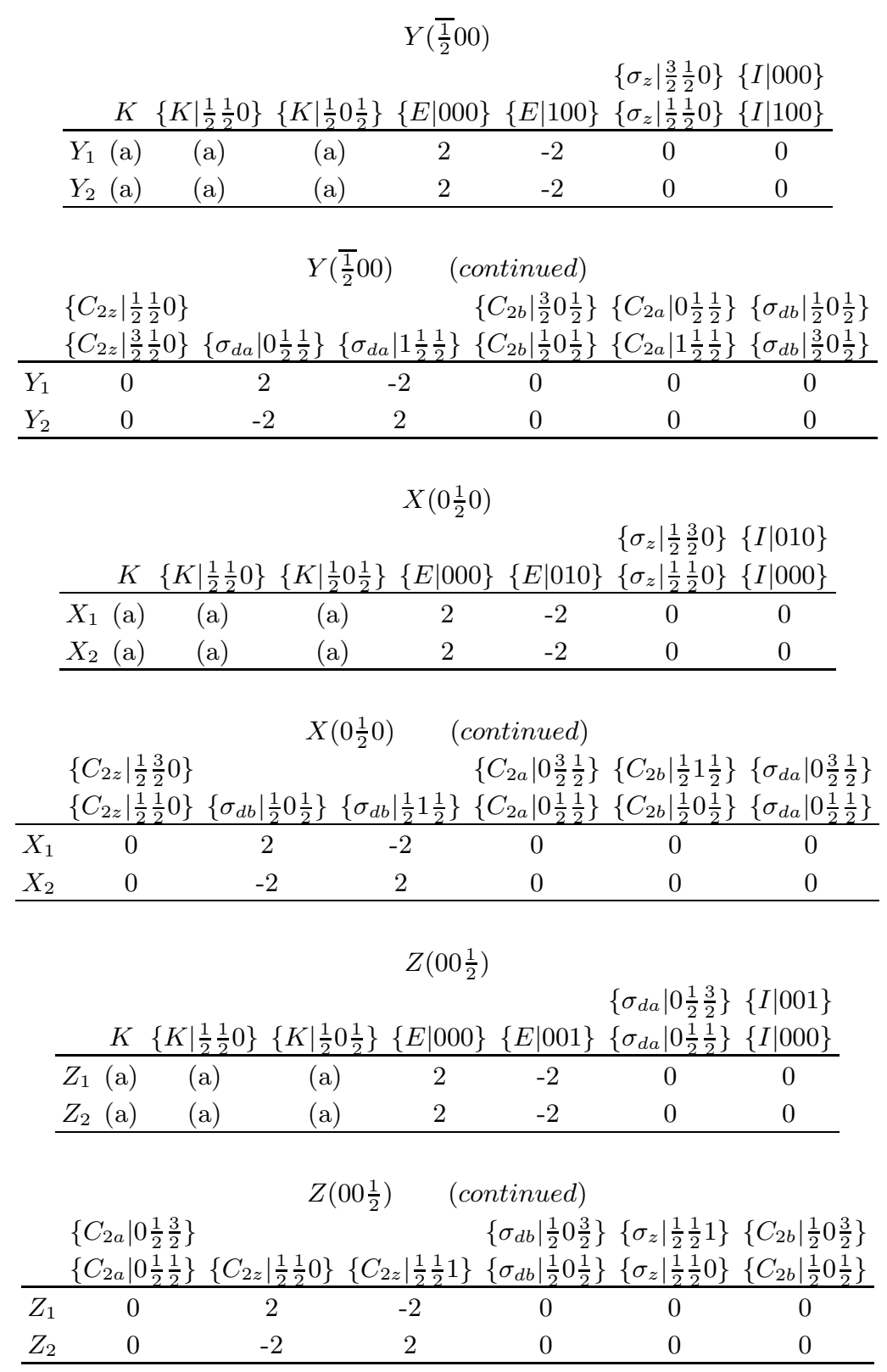


TABLE III: continued

$$
U\left(0 \frac{1}{2} \frac{1}{2}\right)
$$

\begin{tabular}{cccccccc}
\multicolumn{2}{c}{$K$} & $\left\{K \mid \frac{1}{2} \frac{1}{2} 0\right\}$ & $\left\{K \mid \frac{1}{2} 0 \frac{1}{2}\right\}$ & $\{E \mid 000\}$ & $\left\{\sigma_{d a} \mid 0 \frac{1}{2} \frac{3}{2}\right\}$ & $\{E \mid 001\}$ & $\left\{\sigma_{d a} \mid 0 \frac{1}{2} \frac{1}{2}\right\}$ \\
\hline$U_{1}(\mathrm{c})$ & $(\mathrm{a})$ & (c) & 2 & $2 \mathrm{i}$ & -2 & $-2 \mathrm{i}$ \\
$U_{2}(\mathrm{c})$ & $(\mathrm{a})$ & (c) & 2 & $-2 \mathrm{i}$ & -2 & $2 \mathrm{i}$ \\
\hline
\end{tabular}

$$
U\left(0 \frac{1}{2} \frac{1}{2}\right) \quad \text { (continued) }
$$

$\left\{C_{2 z} \mid \frac{1}{2} \frac{1}{2} 0\right\}\left\{\sigma_{d b} \mid \frac{1}{2} 0 \frac{3}{2}\right\}\{I \mid 001\}\left\{C_{2 a} \mid 0 \frac{1}{2} \frac{1}{2}\right\}\left\{\sigma_{z} \mid \frac{1}{2} \frac{1}{2} 0\right\} \quad\left\{C_{2 b} \mid \frac{1}{2} 0 \frac{3}{2}\right\}$

\begin{tabular}{ccccccc} 
& $\left\{C_{2 z} \mid \frac{1}{2} \frac{1}{2} 1\right\}$ & $\left\{\sigma_{d b} \mid \frac{1}{2} 0 \frac{1}{2}\right\}$ & $\{I \mid 000\}$ & $\left\{C_{2 a} \mid 0 \frac{1}{2} \frac{3}{2}\right\}$ & $\left\{\sigma_{z} \mid \frac{1}{2} \frac{1}{2} 1\right\}$ & $\left\{C_{2 b} \mid \frac{1}{2} 0 \frac{1}{2}\right\}$ \\
\hline$U_{1}$ & 0 & 0 & 0 & 0 & 0 & 0
\end{tabular}

\begin{tabular}{lllllll}
$U_{2}$ & 0 & 0 & 0 & 0 & 0 & 0 \\
\hline
\end{tabular}

$$
T\left(\overline{\frac{1}{2}} 0 \frac{1}{2}\right)
$$

\begin{tabular}{ccccccc}
$K$ & $\left\{K \mid \frac{1}{2} \frac{1}{2} 0\right\}$ & $\left\{K \mid \frac{1}{2} 0 \frac{1}{2}\right\}$ & $\{E \mid 000\}$ & $\left\{\sigma_{d b} \mid \frac{1}{2} 0 \frac{1}{2}\right\}$ & $\{E \mid 001\}$ & $\left\{\sigma_{d b} \mid \frac{1}{2} 0 \frac{3}{2}\right\}$ \\
\hline$T_{1}$ (c) & (a) & (a) & 2 & $2 \mathrm{i}$ & -2 & $-2 \mathrm{i}$
\end{tabular}

\begin{tabular}{lllllll}
$T_{2}(\mathrm{c})$ & $(\mathrm{a})$ & (a) & 2 & $-2 \mathrm{i}$ & -2 & $2 \mathrm{i}$ \\
\hline
\end{tabular}

$$
T\left(\frac{1}{2} 0 \frac{1}{2}\right) \quad \text { (continued) }
$$

$\left\{C_{2 z} \mid \frac{1}{2} \frac{1}{2} 1\right\}\left\{\sigma_{d a} \mid 0 \frac{1}{2} \frac{3}{2}\right\}\{I \mid 000\}\left\{C_{2 b} \mid \frac{1}{2} 0 \frac{1}{2}\right\} \quad\left\{\sigma_{z} \mid \frac{1}{2} \frac{1}{2} 0\right\} \quad\left\{C_{2 a} \mid 0 \frac{1}{2} \frac{1}{2}\right\}$

\begin{tabular}{ccccccc} 
& $\left\{C_{2 z} \mid \frac{1}{2} \frac{1}{2} 0\right\}$ & $\left\{\sigma_{d a} \mid 0 \frac{1}{2} \frac{1}{2}\right\}$ & $\{I \mid 001\}$ & $\left\{C_{2 b} \mid \frac{1}{2} 0 \frac{3}{2}\right\}$ & $\left\{\sigma_{z} \mid \frac{1}{2} \frac{1}{2} 1\right\}$ & $\left\{C_{2 a} \mid 0 \frac{1}{2} \frac{3}{2}\right\}$ \\
\hline$T_{1}$ & 0 & 0 & 0 & 0 & 0 & 0 \\
$T_{2}$ & 0 & 0 & 0 & 0 & 0 & 0 \\
\hline
\end{tabular}

$$
S\left(\overline{\frac{1}{2}} \frac{1}{2} 0\right)
$$

$\left\{C_{2 b} \mid \frac{1}{2} 1 \frac{1}{2}\right\}\{I \mid 000\}$

$\frac{K\left\{K \mid \frac{1}{2} \frac{1}{2} 0\right\}\left\{K \mid \frac{1}{2} 0 \frac{1}{2}\right\}\{E \mid 000\}\{E \mid 010\}\left\{C_{2 b} \mid \frac{1}{2} 0 \frac{1}{2}\right\}\{I \mid 010\}}{S_{1} \text { (a) (a) }}$

\begin{tabular}{lllllll}
$S_{2}($ a) & (a) & (c) & 2 & -2 & 0 & 0 \\
\hline
\end{tabular}

$$
S\left(\frac{1}{2} \frac{1}{2} 0\right) \quad(\text { continued })
$$

\begin{tabular}{|c|c|c|c|c|c|c|c|c|c|}
\hline$R_{2}^{-} \quad(\mathrm{c})$ & (a) & (c) & 1 & $-\mathrm{i}$ & -1 & $\mathrm{i}$ & 1 & $-\mathrm{i}$ & -1 \\
\hline$R_{3}^{-} \quad(\mathrm{c})$ & (a) & (c) & 1 & $\mathrm{i}$ & -1 & $-\mathrm{i}$ & -1 & $-\mathrm{i}$ & 1 \\
\hline$R_{4}^{-} \quad(\mathrm{c})$ & (a) & (c) & 1 & $-\mathrm{i}$ & -1 & $\mathrm{i}$ & -1 & $\mathrm{i}$ & 1 \\
\hline$R_{1}^{+} \quad(\mathrm{c})$ & (a) & (c) & 1 & $\mathrm{i}$ & -1 & $-\mathrm{i}$ & 1 & $\mathrm{i}$ & -1 \\
\hline$R_{2}^{+} \quad(\mathrm{c})$ & (a) & (c) & 1 & $-\mathrm{i}$ & -1 & $\mathrm{i}$ & 1 & $-\mathrm{i}$ & -1 \\
\hline$R_{3}^{+} \quad(\mathrm{c})$ & (a) & (c) & 1 & $\mathrm{i}$ & -1 & $-\mathrm{i}$ & -1 & $-\mathrm{i}$ & 1 \\
\hline$R_{4}^{+} \quad(\mathrm{c})$ & (a) & (c) & 1 & $-\mathrm{i}$ & -1 & $\mathrm{i}$ & -1 & $\mathrm{i}$ & 1 \\
\hline
\end{tabular}

$\left\{\sigma_{d b} \mid \frac{1}{2} 0 \frac{1}{2}\right\} \quad\left\{C_{2 a} \mid 0 \frac{1}{2} \frac{1}{2}\right\} \quad\left\{\sigma_{z} \mid \frac{1}{2} \frac{3}{2} 0\right\} \quad\left\{\sigma_{d a} \mid 0 \frac{3}{2} \frac{1}{2}\right\}$

\begin{tabular}{ccccccc} 
& $\left\{\sigma_{d b} \mid \frac{1}{2} 1 \frac{1}{2}\right\}$ & $\left\{C_{2 z} \mid \frac{1}{2} \frac{3}{2} 0\right\}$ & $\left\{C_{2 z} \mid \frac{1}{2} \frac{1}{2} 0\right\}$ & $\left\{C_{2 a} \mid 0 \frac{3}{2} \frac{1}{2}\right\}$ & $\left\{\sigma_{z} \mid \frac{1}{2} \frac{1}{2} 0\right\}$ & $\left\{\sigma_{d a} \mid 0 \frac{1}{2} \frac{1}{2}\right\}$ \\
\hline$S_{1}$ & 0 & 2 & -2 & 0 & 0 & 0 \\
$S_{2}$ & 0 & -2 & 2 & 0 & 0 & 0 \\
\hline
\end{tabular}

$$
R\left(\frac{1}{2} \frac{1}{2} \frac{1}{2}\right)
$$

$K\left\{K \mid \frac{1}{2} \frac{1}{2} 0\right\}\left\{K \mid \frac{1}{2} 0 \frac{1}{2}\right\}\{E \mid 000\}\left\{C_{2 a} \mid 0 \frac{1}{2} \frac{1}{2}\right\}\{E \mid 001\}\left\{C_{2 a} \mid 0 \frac{1}{2} \frac{3}{2}\right\}\left\{C_{2 z} \mid \frac{1}{2} \frac{1}{2} 1\right\}\left\{C_{2 b} \mid \frac{1}{2} 0 \frac{3}{2}\right\}\left\{C_{2 z} \mid \frac{1}{2} \frac{1}{2} 0\right\}$

$$
R\left(\frac{1}{2} \frac{1}{2} \frac{1}{2}\right) \quad(\text { continued })
$$

$\left\{C_{2 b} \mid \frac{1}{2} 0 \frac{1}{2}\right\}\{I \mid 001\}\left\{\sigma_{d a} \mid 0 \frac{1}{2} \frac{3}{2}\right\}\{I \mid 000\}\left\{\sigma_{d a} \mid 0 \frac{1}{2} \frac{1}{2}\right\}\left\{\sigma_{z} \mid \frac{1}{2} \frac{1}{2} 0\right\}\left\{\sigma_{d b} \mid \frac{1}{2} 0 \frac{1}{2}\right\}\left\{\sigma_{z} \mid \frac{1}{2} \frac{1}{2} 1\right\}\left\{\sigma_{d b} \mid \frac{1}{2} 0 \frac{3}{2}\right\}$

\begin{tabular}{lccccccccc}
\hline$R_{1}^{-}$ & $-\mathrm{i}$ & 1 & $\mathrm{i}$ & -1 & $-\mathrm{i}$ & 1 & $\mathrm{i}$ & -1 & $-\mathrm{i}$ \\
$R_{2}^{-}$ & $\mathrm{i}$ & 1 & $-\mathrm{i}$ & -1 & $\mathrm{i}$ & 1 & $-\mathrm{i}$ & -1 & $\mathrm{i}$ \\
$R_{3}^{-}$ & $\mathrm{i}$ & 1 & $\mathrm{i}$ & -1 & $-\mathrm{i}$ & -1 & $-\mathrm{i}$ & 1 & $\mathrm{i}$ \\
$R_{4}^{-}$ & $-\mathrm{i}$ & 1 & $-\mathrm{i}$ & -1 & $\mathrm{i}$ & -1 & $\mathrm{i}$ & 1 & $-\mathrm{i}$ \\
$R_{1}^{+}$ & $-\mathrm{i}$ & -1 & $-\mathrm{i}$ & 1 & $\mathrm{i}$ & -1 & $-\mathrm{i}$ & 1 & $\mathrm{i}$ \\
$R_{2}^{+}$ & $\mathrm{i}$ & -1 & $\mathrm{i}$ & 1 & $-\mathrm{i}$ & -1 & $\mathrm{i}$ & 1 & $-\mathrm{i}$ \\
$R_{3}^{+}$ & $\mathrm{i}$ & -1 & $-\mathrm{i}$ & 1 & $\mathrm{i}$ & 1 & $\mathrm{i}$ & -1 & $-\mathrm{i}$ \\
$R_{4}^{+}$ & $-\mathrm{i}$ & -1 & $\mathrm{i}$ & 1 & $-\mathrm{i}$ & 1 & $-\mathrm{i}$ & -1 & $\mathrm{i}$ \\
\hline
\end{tabular}


TABLE IV: Characters of the small (allowed) double-valued irreducible representations of the space group $I 4 / m m m$ of tetragonal $\mathrm{La}_{2} \mathrm{CuO}_{4}$ in the notation of Table 5.8 in the textbook of Bradley and Cracknell [16].

$\Gamma(000)$ and $Z\left(\frac{1}{2} \frac{1}{2} \frac{1}{2}\right)$

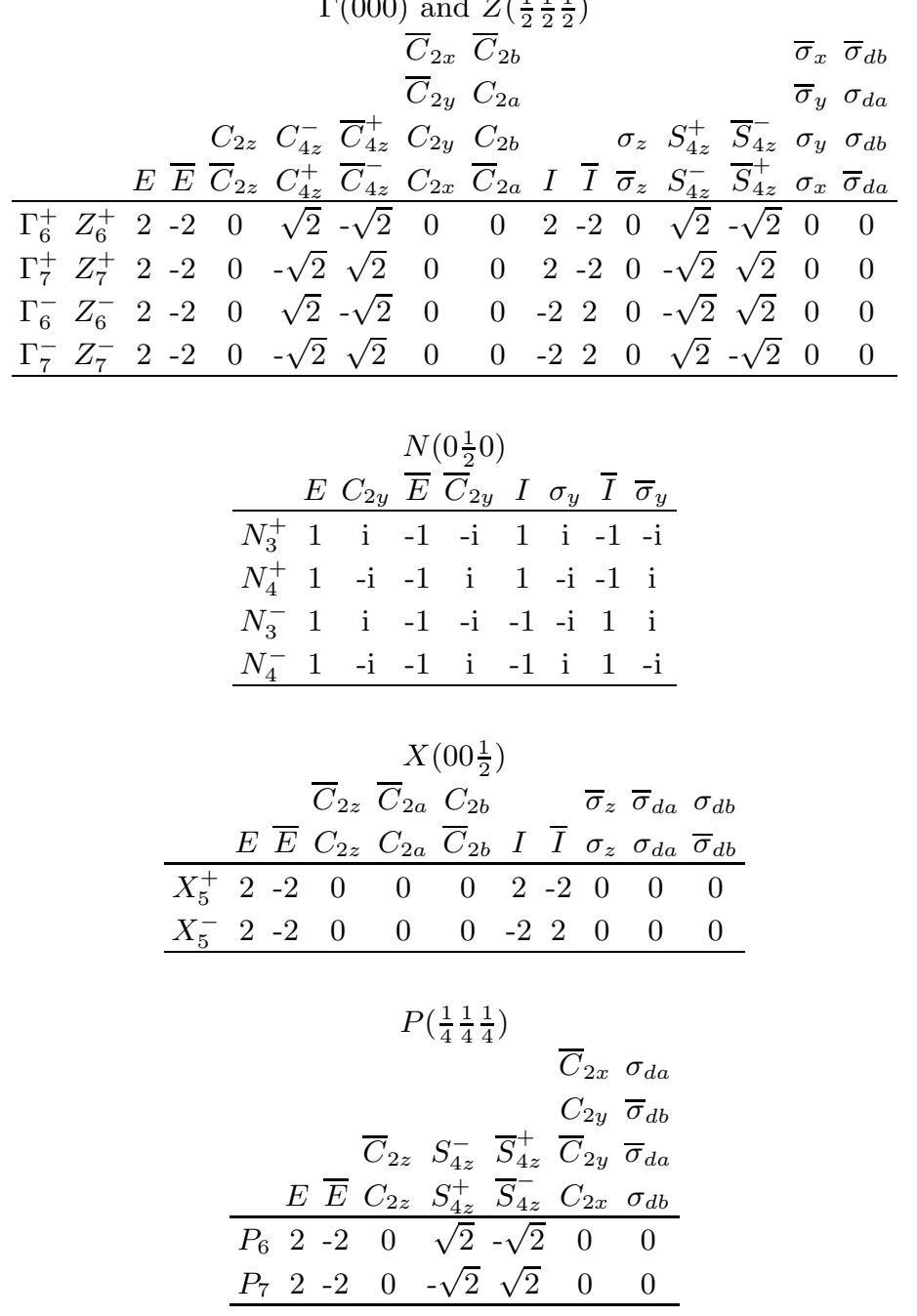


TABLE V: Characters of the small (allowed) double-valued irreducible representations of the space group Cmca of the orthorhombic phase of $\mathrm{La}_{2} \mathrm{CuO}_{4}$ in the notation of Table 5.8 in the textbook of Bradley and Cracknell [16].

$$
\Gamma(000) \text { and } Y\left(\frac{1}{2} \frac{1}{2} 0\right)
$$

$$
\left\{\bar{C}_{2 a} \mid \frac{1}{2} \frac{1}{2} \frac{1}{2}\right\}\left\{\bar{C}_{2 z} \mid \frac{1}{2} \frac{1}{2} \frac{1}{2}\right\}\left\{\bar{C}_{2 b} \mid 000\right\} \quad\left\{\bar{\sigma}_{d a} \mid \frac{1}{2} \frac{1}{2} \frac{1}{2}\right\}\left\{\bar{\sigma}_{z} \mid \frac{1}{2} \frac{1}{2} \frac{1}{2}\right\}\left\{\bar{\sigma}_{d b} \mid 000\right\}
$$
$\{E \mid 000\}\{\bar{E} \mid 000\} \quad\left\{C_{2 a} \mid \frac{1}{2} \frac{1}{2} \frac{1}{2}\right\}\left\{C_{2 z} \mid \frac{1}{2} \frac{1}{2} \frac{1}{2}\right\} \quad\left\{C_{2 b} \mid 000\right\}\{I \mid 000\}\{\bar{I} \mid 000\} \quad\left\{\sigma_{d a} \mid \frac{1}{2} \frac{1}{2} \frac{1}{2}\right\}\left\{\sigma_{z} \mid \frac{1}{2} \frac{1}{2} \frac{1}{2}\right\}\left\{\sigma_{d b} \mid 000\right\}$

\begin{tabular}{|c|c|c|c|c|c|c|c|}
\hline & $\{E \mid 000\}$ & $\{\bar{E} \mid 001\}$ & $Z\left(00 \frac{1}{2}\right.$ & $\left.\frac{1}{2}\right)$ and $T$ & $\begin{array}{l}\left(\frac{\overline{1}}{2} \frac{1}{2} \frac{1}{2}\right) \\
\left\{\bar{C}_{2 a} \mid \frac{1}{2} \frac{1}{2} \frac{1}{2}\right\} \\
\left\{\bar{C}_{2 a} \mid \frac{1}{2} \frac{1}{2} \frac{3}{2}\right\} \\
\left\{C_{2 a} \mid \frac{1}{2} \frac{1}{2} \frac{3}{2}\right\} \\
\left\{C_{2 a} \mid \frac{1}{2} \frac{1}{2} \frac{1}{2}\right\}\end{array}$ & $\begin{array}{l}\left\{\bar{C}_{2 b} \mid 001\right\} \\
\left\{C_{2 b} \mid 000\right\} \\
\end{array}$ & $\begin{array}{l}\left\{C_{2 b} \mid 001\right\} \\
\left\{\bar{C}_{2 b} \mid 000\right\} \\
\end{array}$ \\
\hline$\overline{Z_{3} T_{3}}$ & 2 & 2 & -2 & -2 & 0 & $2 \mathrm{i}$ & $-2 \mathrm{i}$ \\
\hline$Z_{4} T_{4}$ & 2 & 2 & -2 & -2 & 0 & $-2 \mathrm{i}$ & $2 \mathrm{i}$ \\
\hline & $\begin{array}{l}\left\{C_{2 z} \mid \frac{1}{2} \frac{1}{2} \frac{1}{2}\right\} \\
\left\{C_{2 z} \mid \frac{1}{2} \frac{1}{2} \frac{3}{2}\right\} \\
\left\{\bar{C}_{2 z} \mid \frac{1}{2} \frac{1}{2} \frac{3}{2}\right\} \\
\left\{\bar{C}_{2 z} \mid \frac{1}{2} \frac{1}{2} \frac{1}{2}\right\}\end{array}$ & $\begin{array}{r} \\
\} \\
\} \\
\} \\
\} \\
\} I I \mid 001\} \\
\}\{I \mid 000\} \\
\end{array}$ & $\begin{array}{l}\left.\frac{1}{2}\right) \text { and } T \\
\{\bar{I} \mid 000\} \\
\{\bar{I} \mid 001\}\end{array}$ & $\begin{array}{l}\left(\frac{1}{2} \frac{1}{2} \frac{1}{2}\right) \\
\left\{\bar{\sigma}_{d a} \mid \frac{1}{2} \frac{1}{2}\right. \\
\left\{\bar{\sigma}_{d a} \mid \frac{1}{2} \frac{1}{2}\right. \\
\left\{\sigma_{d a} \mid \frac{1}{2} \frac{1}{2}\right. \\
\left\{\sigma_{d a} \mid \frac{1}{2} \frac{1}{2}\right.\end{array}$ & $\begin{aligned} & (\text { continue } \\
\left.\frac{1}{2}\right\} & \\
\left.\frac{3}{2}\right\} & \\
\left.\frac{3}{2}\right\} & \left\{\bar{\sigma}_{d b} \mid 000\right. \\
\left.\frac{1}{2}\right\} & \left\{\sigma_{d b} \mid 000\right.\end{aligned}$ & ) $\begin{array}{l}\left\{\sigma_{d b} \mid 001\right\} \\
)\}\left\{\bar{\sigma}_{d b} \mid 001\right\}\end{array}$ & $\begin{aligned} & \left\{\sigma_{z} \mid \frac{1}{2} \frac{1}{2} \frac{1}{2}\right\} \\
& \left\{\sigma_{z} \mid \frac{1}{2} \frac{1}{2} \frac{3}{2}\right\} \\
\} & \left\{\bar{\sigma}_{z} \mid \frac{1}{2} \frac{1}{2} \frac{3}{2}\right\} \\
\} & \left\{\bar{\sigma}_{z} \mid \frac{1}{2} \frac{1}{2} \frac{1}{2}\right\}\end{aligned}$ \\
\hline$Z_{3} T_{3}$ & 0 & 0 & 0 & 0 & 0 & 0 & 0 \\
\hline$Z_{4} T_{4}$ & 0 & 0 & 0 & 0 & 0 & 0 & 0 \\
\hline
\end{tabular}

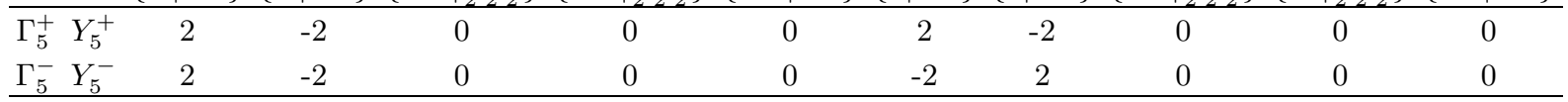

\section{$S\left(0 \frac{1}{2} 0\right)$}

$\{I \mid 010\}\{\bar{I} \mid 000\}\left\{\sigma_{d a} \mid \frac{1}{2} \frac{3}{2} \frac{1}{2}\right\}\left\{\bar{\sigma}_{d a} \mid \frac{1}{2} \frac{1}{2} \frac{1}{2}\right\}\left\{C_{2 a} \mid \frac{1}{2} \frac{3}{2} \frac{1}{2}\right\}\left\{\bar{C}_{2 a} \mid \frac{1}{2} \frac{1}{2} \frac{1}{2}\right\}$

\begin{tabular}{|c|c|c|c|c|c|c|c|c|}
\hline & $\{E \mid 000\}$ & $\left\{\sigma_{d a} \mid \frac{1}{2} \frac{1}{2} \frac{1}{2}\right\}$ & $\{\bar{E} \mid 001\}$ & $\begin{array}{r}R\left(0 \frac{1}{2}\right. \\
\left\{\bar{\sigma}_{d a} \mid \frac{1}{2} \frac{1}{2} \frac{3}{2}\right\}\end{array}$ & $\begin{array}{l}\text { L } \\
\{I \mid 000\}\end{array}$ & $\left\{C_{2 a} \mid \frac{1}{2} \frac{1}{2} \frac{1}{2}\right\}$ & $\{\bar{I} \mid 001\}$ & $\left\{\bar{C}_{2 a} \mid \frac{1}{2} \frac{1}{2} \frac{3}{2}\right\}$ \\
\hline$\overline{R_{3}^{+}}$ & 1 & 1 & 1 & 1 & 1 & 1 & 1 & 1 \\
\hline$R_{4}^{+}$ & 1 & -1 & 1 & -1 & 1 & -1 & 1 & -1 \\
\hline$R_{3}^{-}$ & 1 & 1 & 1 & 1 & -1 & -1 & -1 & -1 \\
\hline$R_{4}^{-}$ & 1 & -1 & 1 & -1 & -1 & 1 & -1 & 1 \\
\hline
\end{tabular}

\begin{tabular}{ccccccccccc} 
& $\{E \mid 000\}$ & $\{E \mid 010\}$ & $\{\bar{E} \mid 010\}$ & $\{\bar{E} \mid 000\}$ & $\{I \mid 000\}$ & $\{\bar{I} \mid 010\}$ & $\left\{\sigma_{d a} \mid \frac{1}{2} \frac{1}{2} \frac{1}{2}\right\}$ & $\left\{\bar{\sigma}_{d a} \mid \frac{1}{2} \frac{3}{2} \frac{1}{2}\right\}$ & $\left\{C_{2 a} \mid \frac{1}{2} \frac{1}{2} \frac{1}{2}\right\}$ & $\left\{\bar{C}_{2 a} \mid \frac{1}{2} \frac{3}{2} \frac{1}{2}\right\}$ \\
\hline$S_{2}$ & 2 & -2 & 2 & -2 & 0 & 0 & 0 & 0 & 0 & 0 \\
\hline
\end{tabular}


TABLE VI: Compatibility relations between the single-valued representations of the space group $I 4 / \mathrm{mmm}$ of tetragonal $\mathrm{La}_{2} \mathrm{CuO}_{4}$ and the single-valued representations of the space group $C m c a$ of the orthorhombic phase of $\mathrm{La}_{2} \mathrm{CuO}_{4}$. For the notations see Tables I and II. The upper row lists small representations at the points of symmetry in the Brillouin zone of $I 4 / \mathrm{mmm}$ and the lower row lists small representations at the related points of symmetry in the Brillouin zone of $\mathrm{Cmca}$ (cf. Fig. 4). The small representations in the same column are compatible in the following sense: Bloch functions which are basis functions of a small representation $R_{i}$ in the upper row also form basis functions of the small representation standing below $R_{i}$

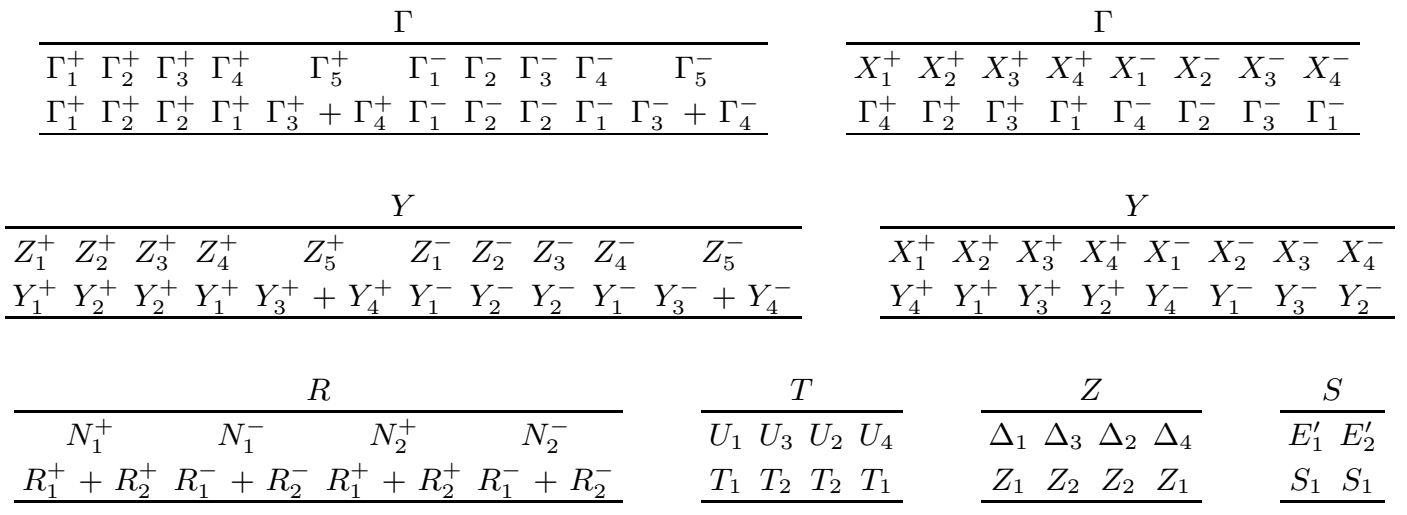

TABLE VII: Compatibility relations between the single-valued representations of the space group $I 4 / \mathrm{mmm}$ of tetragonal $\mathrm{La}_{2} \mathrm{CuO}_{4}$ and the single-valued representations of the space group $P 4 / m n c$ of antiferromagnetic chromium. For the notations see Table I and Table II of Ref. [15]. The upper row lists small representations at the points of symmetry in the Brillouin zone of $I 4 / \mathrm{mmm}$ and the lower row lists small representations at the related points of symmetry in the Brillouin zone of $P 4 / m n c$. The small representations in the same column are compatible in the following sense: Bloch functions which are basis functions of a small representation $R_{i}$ in the upper row also form basis functions of the small representation standing below $R_{i}$.

$\Gamma$

\begin{tabular}{llllllllll}
\hline$\Gamma_{1}^{+}$ & $\Gamma_{2}^{+}$ & $\Gamma_{3}^{+}$ & $\Gamma_{4}^{+}$ & $\Gamma_{5}^{+}$ & $\Gamma_{1}^{-}$ & $\Gamma_{2}^{-}$ & $\Gamma_{3}^{-}$ & $\Gamma_{4}^{-}$ & $\Gamma_{5}^{-}$ \\
$\Gamma_{1}^{+}$ & $\Gamma_{2}^{+}$ & $\Gamma_{3}^{+}$ & $\Gamma_{4}^{+}$ & $\Gamma_{5}^{+}$ & $\Gamma_{1}^{-}$ & $\Gamma_{2}^{-}$ & $\Gamma_{3}^{-}$ & $\Gamma_{4}^{-}$ & $\Gamma_{5}^{-}$ \\
\hline
\end{tabular}

$\Gamma$

\begin{tabular}{llllllllll}
\hline$Z_{1}^{+}$ & $Z_{2}^{+}$ & $Z_{3}^{+}$ & $Z_{4}^{+}$ & $Z_{5}^{+}$ & $Z_{1}^{-}$ & $Z_{2}^{-}$ & $Z_{3}^{-}$ & $Z_{4}^{-}$ & $Z_{5}^{-}$ \\
$\Gamma_{2}^{+}$ & $\Gamma_{1}^{+}$ & $\Gamma_{4}^{+}$ & $\Gamma_{3}^{+}$ & $\Gamma_{5}^{+}$ & $\Gamma_{2}^{-}$ & $\Gamma_{1}^{-}$ & $\Gamma_{4}^{-}$ & $\Gamma_{3}^{-}$ & $\Gamma_{5}^{-}$ \\
\hline
\end{tabular}

\begin{tabular}{lccccccc}
\hline \multicolumn{10}{c}{$M$} \\
\hline$X_{1}^{+}$ & $X_{2}^{+}$ & $X_{3}^{+}$ & $X_{4}^{+}$ & $X_{1}^{-}$ & $X_{2}^{-}$ & $X_{3}^{-}$ & $X_{4}^{-}$ \\
$M_{20}$ & $M_{17}+M_{18}$ & $M_{20}$ & $M_{15}+M_{16}$ & $M_{10}$ & $M_{5}+M_{6}$ & $M_{10}$ & $M_{7}+M_{8}$ \\
\hline
\end{tabular}

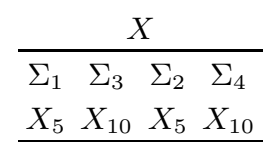

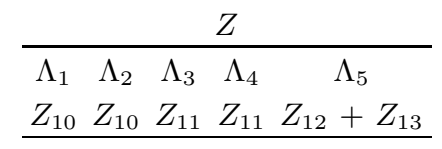

\begin{tabular}{ccccc}
\multicolumn{5}{c}{$A$} \\
\hline$P_{1}$ & $P_{2}$ & $P_{3}$ & $P_{4}$ & $P_{5}$ \\
$A_{11}$ & $A_{11}$ & $A_{10}$ & $A_{10}$ & $A_{12}+A_{13}$ \\
\hline
\end{tabular}

\begin{tabular}{llll}
\multicolumn{4}{c}{$R$} \\
\hline$N_{1}^{+}$ & $N_{1}^{-}$ & $N_{2}^{+}$ & $N_{2}^{-}$ \\
$R_{5}$ & $R_{10}$ & $R_{5}$ & $R_{10}$ \\
\hline
\end{tabular}


TABLE VIII: Compatibility relations between the single-valued representations of the space group $C m c a$ of the orthorhombic phase of $\mathrm{La}_{2} \mathrm{CuO}_{4}$ and the single-valued representations of the space group Pccn of the antiferromagnetic structure of $\mathrm{La}_{2} \mathrm{CuO}_{4}$. For the notations see Tables II and III. The upper row lists small representations at the points of symmetry in the Brillouin zone of $\mathrm{Cmca}$ and the lower row lists small representations at the related points of symmetry in the Brillouin zone of Pccn (cf. Fig. 4). The small representations in the same column are compatible in the following sense: Bloch functions which are basis functions of a small representation $R_{i}$ in the upper row also form basis functions of the small representation standing below $R_{i}$.

\begin{tabular}{|c|c|}
\hline$\Gamma$ & $\Gamma$ \\
\hline 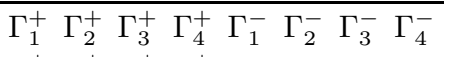 & $Y_{1}^{+} Y_{2}^{+} Y_{3}^{+} Y_{4}^{+} Y_{1}^{-} Y_{2}^{-} Y_{3}^{-} Y_{4}^{-}$ \\
\hline 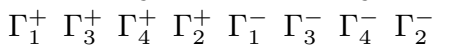 & $\begin{array}{cccccccc}\Gamma_{3}^{+} & \Gamma_{1}^{+} & \Gamma_{2}^{+} & \Gamma_{4}^{+} & \Gamma_{3}^{-} & \Gamma_{1}^{-} & \Gamma_{2}^{-} & \Gamma_{4}^{-}\end{array}$ \\
\hline
\end{tabular}

\begin{tabular}{|c|c|c|c|c|}
\hline$X$ & $X$ & $T$ & $S$ & $R$ \\
\hline$Z_{1} \quad Z_{2}$ & $T_{1} T_{2}$ & $S_{1}$ & $A_{1}$ & $R_{2}^{+}$ \\
\hline$X_{1} \quad X_{2}$ & $X_{2} X_{1}$ & $\underline{T_{1}+T_{2}}$ & $\underline{S_{1}+S_{2}}$ & $R_{1}^{+}+R_{3}^{+} R_{2}^{+}+R_{4}^{+} R_{2}^{-}+R_{4}^{-} R_{1}^{-}+R_{3}^{-}$ \\
\hline & & $Y$ & $U$ & $Z$ \\
\hline & & $\Sigma_{1} \quad \Sigma_{3} \quad \Sigma_{2} \quad \Sigma_{4}$ & $B_{1} B_{2} B_{3} \quad B_{4}$ & $\Delta_{1} \Delta_{3} \Delta_{2} \Delta_{4}$ \\
\hline & & $\begin{array}{llll}Y_{1} & Y_{2} & Y_{1} & Y_{2} \\
\end{array}$ & $\begin{array}{llll}U_{1} & U_{2} & U_{2} & U_{1} \\
\end{array}$ & $\begin{array}{llll}Z_{1} & Z_{1} & Z_{2} & Z_{2} \\
\end{array}$ \\
\hline
\end{tabular}

TABLE IX: Compatibility relations between the single-valued (upper row) and double-valued (lower row) representations of the space group $I 4 / \mathrm{mmm}$ of tetragonal $\mathrm{La}_{2} \mathrm{CuO}_{4}$. For the notations see Tables I and IV. The small representations in the same column are compatible in the following sense: spin-dependent Bloch functions which are basis functions of the small representation $R_{i} \times D_{1 / 2}$ (with $R_{i}$ standing in the upper row) also form basis functions of the double-valued small representation standing below $R_{i}$. $D_{1 / 2}$ denotes the two-dimensional double-valued representation of the three-dimensional rotation group $O(3)$ (given, e.g., in Table 6.1 of Ref. [16]).

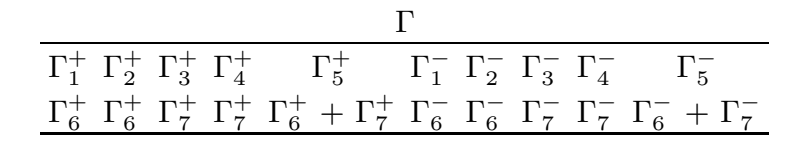

\begin{tabular}{cccc}
\multicolumn{6}{c}{$N$} \\
\hline $\begin{array}{c}N_{1}^{+} \\
N_{3}^{+}+N_{4}^{+}\end{array}$ & $N_{3}^{-}+N_{4}^{-}$ & $N_{3}^{+}+N_{4}^{+}$ & $N_{3}^{-}+N_{4}^{-}$ \\
\hline
\end{tabular}

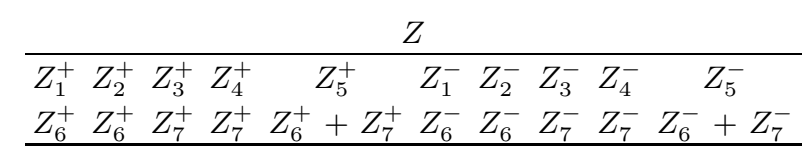

\begin{tabular}{lllll}
\multicolumn{4}{c}{$P$} \\
\hline$P_{1}$ & $P_{2}$ & $P_{3}$ & $P_{4}$ & $P_{5}$ \\
$P_{6}$ & $P_{6}$ & $P_{7}$ & $P_{7}$ & $P_{6}+P_{7}$ \\
\hline
\end{tabular}

TABLE X: Compatibility relations between the single-valued (upper row) and double-valued (lower row) representations of the space group $C m c a$ of the orthorhombic phase of $\mathrm{La}_{2} \mathrm{CuO}_{4}$. For the notations see Tables II and V. The small representations in the same column are compatible in the following sense: spin-dependent Bloch functions which are basis functions of the small representation $R_{i} \times D_{1 / 2}$ (with $R_{i}$ standing in the upper row) also form basis functions of the double-valued representation standing below $R_{i} . D_{1 / 2}$ denotes the two-dimensional double-valued representation of the three-dimensional rotation group $O(3)$ (given, e.g., in Table 6.1 of Ref. [16]).

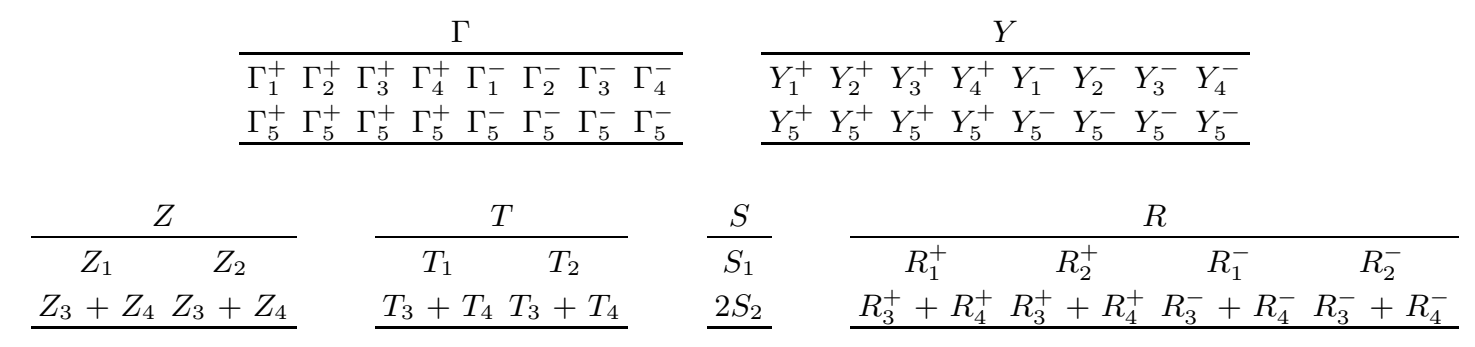


TABLE XI: Single-valued small representations (in the notation given in Table I) of all the single bands in the space group $I 4 / \mathrm{mmm}$ of tetragonal $\mathrm{La}_{2} \mathrm{CuO}_{4}$ with symmetry-adapted and optimally localizable Wannier functions centered at the $\mathrm{Cu}$ atoms.

\begin{tabular}{lllllll}
\hline Band 1 & $\Gamma_{1}^{+}$ & $Z_{1}^{+}$ & $N_{1}^{+}$ & $X_{1}^{+}$ & $P_{1}$ \\
Band 2 & $\Gamma_{2}^{+}$ & $Z_{2}^{+}$ & $N_{2}^{+}$ & $X_{3}^{+}$ & $P_{2}$ \\
Band 3 & $\Gamma_{3}^{+}$ & $Z_{3}^{+}$ & $N_{1}^{+}$ & $X_{3}^{+}$ & $P_{3}$ \\
Band 4 & $\Gamma_{4}^{+}$ & $Z_{4}^{+}$ & $N_{2}^{+}$ & $X_{1}^{+}$ & $P_{4}$ \\
Band 5 & $\Gamma_{1}^{-}$ & $Z_{1}^{-}$ & $N_{1}^{-}$ & $X_{1}^{-}$ & $P_{3}$ \\
Band 6 & $\Gamma_{2}^{-}$ & $Z_{2}^{-}$ & $N_{2}^{-}$ & $X_{3}^{-}$ & $P_{4}$ \\
Band 7 & $\Gamma_{3}^{-}$ & $Z_{3}^{-}$ & $N_{1}^{-}$ & $X_{3}^{-}$ & $P_{1}$ \\
Band 8 & $\Gamma_{4}^{-}$ & $Z_{4}^{-}$ & $N_{2}^{-}$ & $X_{1}^{-}$ & $P_{2}$ \\
\hline
\end{tabular}

TABLE XII: (Double-valued) small representations (in the notation given in Table IV) of all the superconducting bands in the space group $I 4 / \mathrm{mmm}$ of tetragonal $\mathrm{La}_{2} \mathrm{CuO}_{4}$ with symmetry-adapted and optimally localizable spin-dependent Wannier functions centered at the $\mathrm{Cu}$ atoms.

\begin{tabular}{lllllll}
\hline Band 1 & $\Gamma_{6}^{+}$ & $Z_{6}^{+}$ & $N_{3}^{+}+N_{4}^{+}$ & $X_{5}^{+}$ & $P_{6}$ \\
Band 2 & $\Gamma_{7}^{+}$ & $Z_{7}^{+}$ & $N_{3}^{+}+N_{4}^{+}$ & $X_{5}^{+}$ & $P_{7}$ \\
Band 3 & $\Gamma_{6}^{-}$ & $Z_{6}^{-}$ & $N_{3}^{-}+N_{4}^{-}$ & $X_{5}^{-}$ & $P_{7}$ \\
Band 4 & $\Gamma_{7}^{-}$ & $Z_{7}^{-}$ & $N_{3}^{-}+N_{4}^{-}$ & $X_{5}^{-}$ & $P_{6}$ \\
\hline
\end{tabular}

TABLE XIII: (Double-valued) small representations (in the notation given in Table V) of all the superconducting bands in the space group $\mathrm{Cmca}$ of the orthorhombic phase of $\mathrm{La}_{2} \mathrm{CuO}_{4}$ with symmetry-adapted and optimally localizable spin-dependent Wannier functions centered at the $\mathrm{Cu}$ atoms.

\begin{tabular}{lllllll}
\hline Band 1 & $2 \Gamma_{5}^{+}$ & $2 Y_{5}^{+}$ & $Z_{3}+Z_{4}$ & $T_{3}+T_{4}$ & $2 S_{2}$ & $2 R_{3}^{+}+2 R_{4}^{+}$ \\
Band 2 & $2 \Gamma_{5}^{-}$ & $2 Y_{5}^{-}$ & $Z_{3}+Z_{4} T_{3}+T_{4}$ & $2 S_{2}$ & $2 R_{3}^{-}+2 R_{4}^{-}$ \\
\hline
\end{tabular}

TABLE XIV: (Single-valued) small representations (in the notation given in Table II) of all the neutral bands in the space group Cmca of the orthorhombic phase of $\mathrm{La}_{2} \mathrm{CuO}_{4}$ with symmetry-adapted and optimally localizable Wannier functions centered at the $\mathrm{Cu}$ atoms. The Wannier functions may be constructed so that either they are symmetry-adapted to the magnetic group $M_{1}=C m c a+\left\{K \mid \frac{1}{2} \frac{1}{2} \frac{1}{2}\right\} C m c a$ or to the grey magnetic group $M_{2}=C m c a+K \cdot C m c a$ because Eq. (B16) is satisfied in both cases. However, an antiferromagnetic structure with the magnetic group $M_{1}$ is unstable, see Appendix A. Hence, the listed bands are neutral bands with Wannier functions adapted to $M_{2}$.

\begin{tabular}{lllllll}
\hline Band 1 & $\Gamma_{1}^{+}+\Gamma_{4}^{+} Y_{1}^{+}+Y_{4}^{+} Z_{1} T_{1} S_{1} R_{1}^{+}+R_{2}^{+}$ \\
Band 2 & $\Gamma_{2}^{+}+\Gamma_{3}^{+} Y_{2}^{+}+Y_{3}^{+} Z_{2} T_{2}$ & $S_{1}$ & $R_{1}^{+}+R_{2}^{+}$ \\
Band 3 & $\Gamma_{1}^{-}+\Gamma_{4}^{-}$ & $Y_{1}^{-}+Y_{4}^{-}$ & $Z_{2}$ & $T_{2}$ & $S_{1}$ & $R_{1}^{-}+R_{2}^{-}$ \\
Band 4 & $\Gamma_{2}^{-}+\Gamma_{3}^{-}$ & $Y_{2}^{-}+Y_{3}^{-}$ & $Z_{1}$ & $T_{1}$ & $S_{1}$ & $R_{1}^{-}+R_{2}^{-}$ \\
\hline
\end{tabular}

TABLE XV: (Single-valued) small representations (in the notation of Ref. [15]) of all the antiferromagnetic bands in the space group $P 4 / m n c=D_{4 h}^{6}$ (128) of antiferromagnetic Cr with symmetry-adapted and optimally localizable Wannier functions centered at the $\mathrm{Cu}$ atoms. This table has already been published in Ref. [20].

\begin{tabular}{lllllll}
\hline Band 1 & $\Gamma_{1}^{+}+\Gamma_{2}^{+}$ & $X_{5}$ & $M_{20}$ & $A_{11}$ & $Z_{10}$ & $R_{5}$ \\
Band 2 & $\Gamma_{3}^{+}+\Gamma_{4}^{+}$ & $X_{5}$ & $M_{20}$ & $A_{10}$ & $Z_{11}$ & $R_{5}$ \\
Band 3 & $\Gamma_{1}^{-}+\Gamma_{2}^{-}$ & $X_{10}$ & $M_{10}$ & $A_{10}$ & $Z_{10}$ & $R_{10}$ \\
Band 4 & $\Gamma_{3}^{-}+\Gamma_{4}^{-}$ & $X_{10}$ & $M_{10}$ & $A_{11}$ & $Z_{11}$ & $R_{10}$ \\
\hline
\end{tabular}


TABLE XVI: (Single-valued) small representations (in the notation given in Table III) of all the antiferromagnetic bands in the space group Pccn of the (experimentally established) antiferromagnetic structure of $\mathrm{La}_{2} \mathrm{CuO}_{4}$ with symmetry-adapted and optimally localizable Wannier functions centered at the $\mathrm{Cu}$ atoms. An antiferromagnetic structure with the magnetic group $M=P c c n+\left\{K \mid \frac{1}{2} \frac{1}{2} 0\right\}$ Pccn can be stable, see Appendix A.

Band $1 \quad \Gamma_{1}^{+}+\Gamma_{2}^{+}+\Gamma_{3}^{+}+\Gamma_{4}^{+} Y_{1}+Y_{2} X_{1}+X_{2} Z_{1}+Z_{2} U_{1}+U_{2} T_{1}+T_{2} S_{1}+S_{2} R_{1}^{+}+R_{2}^{+}+R_{3}^{+}+R_{4}^{+}$

$\underline{\text { Band } 2 \Gamma_{1}^{-}+\Gamma_{2}^{-}+\Gamma_{3}^{-}+\Gamma_{4}^{-} Y_{1}+Y_{2} X_{1}+X_{2} Z_{1}+Z_{2} U_{1}+U_{2} T_{1}+T_{2} S_{1}+S_{2} R_{1}^{-}+R_{2}^{-}+R_{3}^{-}+R_{4}^{-}}$ 